bioRxiv preprint doi: https://doi.org/10.1101/2020.07.05.179689. this version posted November 24,2020 . The copyright holder for this preprint (which was not certified by peer review) is the author/funder, who has granted bioRxiv a license to display the preprint in perpetuity. It is made available under aCC-BY-NC 4.0 International license.

\title{
Isolation and characterisation of Klebsiella phages for phage therapy
}

Eleanor Townsend ${ }^{1}$, Lucy Kelly ${ }^{1}$, Lucy Gannon ${ }^{2}$, George Muscatt ${ }^{1}$, Rhys Dunstan ${ }^{3}$, Slawomir Michniewski ${ }^{1}$, Hari Sapkota ${ }^{2}$, Saija J Kiljunen ${ }^{4}$, Anna Kolsi ${ }^{4}$, Mikael Skurnik ${ }^{4,5}$, Trevor Lithgow ${ }^{3}$, Andrew D. Millard ${ }^{2}$, Eleanor Jameson ${ }^{1}$

${ }^{1}$ School of Life Sciences, Gibbet Hill Campus, The University of Warwick, Coventry, CV4 7AL ${ }^{2}$ Department of Genetics, University of Leicester, Leicester, LE1 7RH

${ }^{3}$ Monash Biomedicine Discovery Institute, Monash University, 3800 Melbourne, Australia

${ }^{4}$ Department of Bacteriology and Immunology, Human Microbiome Research Program, Faculty of Medicine, University of Helsinki, 00014, Finland

${ }^{5}$ Division of Clinical Microbiology, Helsinki University Hospital, HUSLAB, 00290 Helsinki, Finland

Corresponding author: Eleanor Jameson, Eleanor.Jameson@warwick.ac.uk, M130, School of Life Sciences, Gibbet Hill Campus, The University of Warwick, Coventry, CV4 7AL

\section{Keywords:}

Klebsiella, bacteriophage, phage, phage therapy, antimicrobial resistance, antibiotics, nosocomial infection, characterisation, virulence 
bioRxiv preprint doi: https://doi.org/10.1101/2020.07.05.179689; this version posted November 24,2020 . The copyright holder for this preprint (which was not certified by peer review) is the author/funder, who has granted bioRxiv a license to display the preprint in perpetuity. It is made available under aCC-BY-NC 4.0 International license.

\begin{abstract}
Klebsiella is a clinically important pathogen causing a variety of antimicrobial resistant infections in both community and nosocomial settings, particularly pneumonia, urinary tract infection and sepsis. Bacteriophage (phage) therapy is being considered as a primary option for the treatment of drugresistant infections of these types. We report the successful isolation and characterisation of 30 novel, genetically diverse Klebsiella phages. The isolated phages span six different phage families and nine genera, representing both lysogenic and lytic lifestyles. Individual Klebsiella phage isolates infected up to 11 of the 18 Klebsiella capsule types tested, and all 18 capsule-types were infected by at least one of the phages. Of the Klebsiella-infecting phages presented in this study, the lytic phages are most suitable for phage therapy, based on their broad host range, high virulence, short lysis period and given that they encode no known toxin or antimicrobial resistance genes. Importantly, when applied alone, none of the characterised phages were able to suppress the growth of Klebsiella for more than 12 hours, with some phages only able to suppress growth for 3 hours, likely due to inherent ease of Klebsiella to generate spontaneous phage-resistant mutants. This indicates that for successful phage therapy, a cocktail of multiple phages would be necessary to treat Klebsiella infections.
\end{abstract}




\section{Background}

Bacteria of the Klebsiella genus are able to cause a variety of infections in both community and nosocomial settings including pneumonia, urinary tract infection (UTI), sepsis, wound infection, and infections in vulnerable populations including neonates and intensive care patients ${ }^{1}$. Klebsiella is also one of the most numerous secondary infection agents in COVID-19 patients, particularly those who have undergone ventilation ${ }^{2-4}$. Additionally, the sub-clinical carriage of Klebsiella is linked to cardiovascular disease ${ }^{5,6}$ and inflammatory bowel disease ${ }^{7}$. Klebsiella pneumoniae is the most highly reported problematic pathogen from the genus; it has given rise to hypervirulent clones with extended virulence factors. Other Klebsiella species, including $K$. oxytoca $^{8}$ and $K$. variicola ${ }^{9}$, are emerging pathogens, causing infections in immunocompromised patients. Whilst the arsenal of virulence factors make Klebsiella efficient pathogens, it is the high prevalence of antimicrobial resistance (AMR) mechanisms that complicates treatment and leads to high mortality ${ }^{10}$.

AMR represents a current threat to global health and security, fueled by our intensive use of antibiotics in both medicine and agriculture. Klebsiella is termed an ESKAPE pathogen on the World Health Organisation priority pathogens list ${ }^{11}$, gaining and transferring AMR genes, particularly in health-care settings ${ }^{12}$. The prevalence of resistance in Klebsiella has increased exponentially to most available antimicrobial drugs, and cases of pan-resistant Klebsiella are are now common around the world ${ }^{13-16}$. This poses a difficulty in treating these infections with existing antibiotics available in clinical settings ${ }^{17}$ and yet there are also major issues associated with vaccine development to prevent these infections ${ }^{18}$. The economic cost of Klebsiella outbreaks are high; in 2015 a Dutch hospital estimated the cost of an outbreak of multidrug-resistant (MDR) $K$. pneumoniae to be $\$ 804,263^{19}$. Alongside the economic consequences of these MDR Klebsiella infections, there is an increased risk of mortality ${ }^{19,20}$. One potential alternative treatment to antibiotics are bacteriophages (phages) ${ }^{21}$.

Phages are natural killers of bacteria and phage therapy is emerging as a potential weapon against MDR bacterial infections ${ }^{22,23}$. Phage therapy depends on preparedness, particularly in having a real or virtual "biobank" of phages suited for killing the bacteria responsible for common, AMR infections. The discovery of phages for therapeutic use is realistic given the low-costs of modern sequencing technologies and the classic technologies for characterization of phage virions ${ }^{24}$. Pre-therapeutic characterisation of phages is essential to mitigate side effects in patients, gain access to purified active phages in a timely manner, establish the best mode of delivery and the level of inactivation of phages by the immune system. It has been highlighted how awareness of broad aspects of phage biology can minimize potential side-effects during therapy ${ }^{25}$. Ultimately, these hurdles can be overcome with detailed understanding and characterisation of prospective phage genomes, structure and function.

In this paper we use genomic and imaging technologies to characterise novel phages isolated against clinical and environmental Klebsiella spp. Phages were discovered in a range of environmental scenarios including rivers, ponds, estuaries, canals, slurry, and sewage. The newly isolated phages were characterised using a combination of traditional and genomic approaches to understand their infection cycle, host range, and gene content. We present phages with siphovirus, myovirus, podovirus and inovirus morphologies, spanning six phage families and nine genera, of which the majority have lytic lifestyles. A number of these phages have potential use in phage therapy. 


\section{Materials and Methods}

Bacterial Strains and Culture Conditions

The Klebsiella strains used in this work are listed in table 1. All culturing in liquid medium was performed with shaking $(150 \mathrm{rpm})$ at $37^{\circ} \mathrm{C}$. All culturing was carried out in Lysogeny broth (LB), with the addition of $5 \mathrm{mM} \mathrm{CaCl}_{2}$ and $5 \mathrm{mM} \mathrm{MgCl}_{2}$ when culturing phages. The Klebsiella were originally isolated from clinical samples, except for two which were of environmental origin (table 1). The strains represented six species: $K$. pneumoniae, $K$. oxytoca, $K$. quasipneumoniae, $K$. variicola, $K$. michagenesis and $K$. aerogenes.

\section{Klebsiella capsule typing}

Kaptive Web was used and determined that the 24 Klebsiella strains belonged to 18 different capsule types (table 1). Three K. pneumoniae strains were identified as capsule type KL2, while two Klebsiella $\mathrm{sp}$. were each typed as O1v1, OL104 and KL14. All other capsule types were unique, $K$. aerogenes 30053 could not be typed.

\section{Phage Isolation}

Phages were isolated from water samples from various sources, listed in table 2. Phages were named using the ICTV binomial system of viral nomenclature ${ }^{26}$. The water samples were filtered through 0.2 $\mu \mathrm{m}$ pore size syringe filters to remove debris and bacteria. Phages were then isolated by enrichment: $2.5 \mathrm{~mL}$ of filtered water sample was added to $2.5 \mathrm{ml}$ nutrient broth, containing $5 \mathrm{mM} \mathrm{CaCl}_{2}$ and $5 \mathrm{mM}$ $\mathrm{MgCl}_{2}$, and inoculated with $50 \mu \mathrm{L}$ of overnight-grown Klebsiella. This enrichment culture was then incubated overnight at $37{ }^{\circ} \mathrm{C}$, centrifuged, and the supernatant filtered through a $0.2 \mu \mathrm{m}$ pore size filter to remove cells. This filtrate was serially diluted down to $10^{-11}$ in LB and used in an overlay agar plaque assay. Briefly, $50 \mu \mathrm{l}$ of each serial dilution was mixed with $0.5 \mathrm{~mL}$ of a single Klebsiella strain in the logarithmic growth phase $\left(\mathrm{OD}_{600} 0.2\right)$ and incubated at room temperature for $5 \mathrm{~min}$. To each serial dilution/cell mix, $2.5 \mathrm{~mL}$ of cooled, molten LB agar ( $0.4 \%$ weight/volume) was added and mixed by swirling. The molten agar mix was poured onto $1 \%$ LB agar plates. All overlay agar plates were allowed to set, then inverted and incubated overnight at $37^{\circ} \mathrm{C}$. From the plaque assay plates single plaques were identified, picked using a pipette tip, mixed with $50 \mu \mathrm{L}$ of $\mathrm{LB}$, and filtered through a 0.22 $\mu \mathrm{m}$ spin filter (Costar Spin-X, Corning, UK). The filtrate underwent two further rounds of plaque assay to ensure that phage isolates were the result of a single clonal phage.

\section{DNA Extraction}

DNA was extracted using the Phenol Chloroform Method ${ }^{27}$. Briefly, phage lysates were concentrated using a protein column with a $30 \mathrm{kDa}$ cut-off. $750 \mu \mathrm{L}$ of concentrated phage was treated with DNase I and Proteinase K, prior to phenol-chloroform, then overnight precipitation with ammonium acetate and ethanol at $-20^{\circ} \mathrm{C}$. The DNA was resuspended in $50 \mu \mathrm{L}$ of molecular grade water.

For bacteriophage which had a high background level of protein contamination from the host strain, Norgen Phage DNA Isolation Kit was used following the manufacturer's instructions. To assess the quantity and quality of the isolated DNA for sequencing, both a spectrophotometer-based method and Qubit were used.

\section{Genome Sequencing}

Sequencing was performed by MicrobesNG (Birmingham, UK), briefly; genomic DNA libraries were prepared using Nextera XT Library Prep Kit (Illumina, San Diego, USA) following the manufacturer's protocol with modifications: $2 \mathrm{ng}$ of DNA were used as the input, and a PCR elongation time of $1 \mathrm{~min}$. DNA quantification and library preparation were carried out on a Hamilton Microlab STAR automated liquid handling system. Pooled libraries were quantified using the Kapa Biosystems Library Quantification Kit for Illumina, on a Roche light cycler 96 qPCR machine. Libraries were sequenced on the Illumina HiSeq using a 250 bp paired end protocol. 


\section{Bioinformatics}

Contig and genome assembly was carried out by MicrobesNG; reads were trimmed with Trimmomatic 0.30 , sliding window quality cutoff of $Q 15^{28}$, SPAdes (v3.7) was used for de novo assembly ${ }^{29}$. Genomes were annotated using Prokka ${ }^{30}$, a custom database of phage genomes extracted from Genbank was used as previously described ${ }^{31}$. The capsule types of the Klebsiella strain genomes were predicted using Kaptive ${ }^{32}$.

To determine the taxonomy of our phages, the genomes were added to VIPtree ${ }^{33}$. Phage isolate genomes were then subject to the BLASTn and tBLASTn against NCBI. Average nucleotide identity (ANI) of the closest genomes identified were compared to our phages using orthoANI (https://www.ezbiocloud.net/tools/ani) ${ }^{34}$. Genomes with an ANI $>95 \%$ were designated as the same species ${ }^{35}$. For genus-level clustering a shared protein network analysis was performed using vConTACT2 (v0.9.13) ${ }^{36}$ with all phage genomes available at the time (May 2020) ${ }^{31}$. The resulting network graph was visualised and annotated within Cytoscape (v3.8.0) ${ }^{37}$. Finally, multiple sequence alignments were performed using MAFFT (v7.271) ${ }^{38}$ on the DNA polymerase, large terminase subunit and major capsid proteins of each phage isolate genus with the most closely related phage proteins, identified. Phylogenetic trees were constructed with RaxML (v8.2.4) ${ }^{39}$ with 1000 bootstrap calculations using the GAMMA model of heterogeneity and the maximum-likelihood method based on the JTT substitution matrix. Subsequent trees were visualized and annotated in R (v3.6.1) using the ggtree (v1.16.6) ${ }^{40,41}$ and phytools (v0.7-70) packages ${ }^{42}$.

Putative structural genes from the phage isolates were analysed for the presence of predicted depolymerase, enzymatic domains or features common to characterised depolymerase proteins. Each predicted gene product was analysed by BLASTP (v2.10.0;

https://blast.ncbi.nlm.nih.gov/Blast.cgi), Pfam HMMER (v3.3; https://www.ebi.ac.uk/Tools/hmmer/) and HHpred (v33.1; https://toolkit.tuebingen.mpg.de/) using the default settings. Sequences from biochemically characterised depolymerase proteins that target Klebsiella spp. (table S1) or the putative depolymerases from our phage isolates (table S2) were used for analysis. Sequences were aligned with Muscle (v3.8.31) ${ }^{43}$ using SeaView (v4) ${ }^{44}$. Phylogenetic tree construction was performed with MegaX ${ }^{45}$ with 500 bootstrap calculations using the LG model. Tree topology searches were performed using a combination of $\mathrm{NNI}$ and $\mathrm{NJ} / \mathrm{BioNJ}$. The tree was subsequently visualised and annotated using iTOL(v4) ${ }^{46}$.

\section{Host Range Testing}

Host range was tested by plating $5 \mu \mathrm{L}$ of phage stock serial dilutions on to bacterial lawn, in $0.4 \%$ overlay agar. Zones of clearing, indicating cell lysis were recorded: visible plaques, clearing at high phage titres, reduced lawn at high phage titres, turbid lawn with neat phage, or no effect.

\section{Plaque Formation and Morphology}

Pure phage stocks were plated using the overlay agar plaque assay method, as described above, on their isolation host. Plates were incubated overnight at $37^{\circ} \mathrm{C}$ to allow plaques to form. Plaque morphology was noted and photographs taken.

\section{Transmission Electron Microscopy}

Pure phage stocks were imaged on formvar/carbon-coated copper grids (Agar Scientific Ltd, UK). To prepare grids for transmission electron microscope (TEM), the grids were glow-discharged for $1 \mathrm{~min}$ under vacuum. Following this, $5 \mu \mathrm{L}$ of phage stock was applied to grid and incubated for $1.5 \mathrm{~min}$ at room temperature. The grid was then blotted to remove excess liquid. A drop of $2 \%$ uranyl acetate was then applied to the grid and incubated for $1 \mathrm{~min}$, before blotting off. The uranyl acetate staining was repeated four times. The grid was allowed to air dry after the final blot. Stained phage grids were imaged on a JEOL 2100Plus TEM. The morphology of the phage particles was visualised in ImageJ, 
phage particle capsids and tails were measured using the measure function. A median of thirty phage particles were used to calculate phage measurements.

Lysis Period

Klebsiella cultures in the exponential growth phase were normalised to an OD $600 \mathrm{~nm}$ of 0.2 , using a spectrophotometer and phage lysates were diluted 1:4. The OD $600 \mathrm{~nm}$ was measured every 5 min for $16 \mathrm{hr}$. Growth was compared to a positive control culture without the addition of phages. The lysis period was calculated by measuring the time from phage addition to a drop in culture OD $600 \mathrm{~nm}$, relative to the positive control, indicating bacterial cell lysis.

\section{Virulence Index}

The two virulence metrics the virulence Index (VP) and MV50 were calculated based on the protocol described by Storms and others ${ }^{47}$. Briefly, bacterial cultures were grown to exponential phase and then adjusted (as described for lysis period) to an optical density equivalent to $1 \times 10^{8} \mathrm{cfu} / \mathrm{mL}$. In a 96well plate, phages were serially diluted from $1 \times 10^{8} \mathrm{pfu} / \mathrm{mL}$ to $10 \mathrm{pfu} / \mathrm{mL}$ in $100 \mu \mathrm{L}$ volumes. A bacterial culture was then added in equal volume $(100 \mu \mathrm{L})$ to the phage dilution, resulting in multiplicity of infections (MOIs) from 1 to $10^{-7}$. The optical density of the 96 -well plate was read at 600 $\mathrm{nm}$ at 5 min intervals for 18 hours. To calculate virulence indices, the area under the curve was calculated for both the bacterial only control and at each phage $\mathrm{MOI}$, from time of initial infection until the exponential growth stage. The VP at each MOI was calculated following the method described ${ }^{47}$ using RStudio (version 1.1.463). The two virulence metrics capture different aspects of infection: VP is a quantified measure of the virulence of a phage against a bacterial host on a scale of 0-1 (from 0 , no reduction in bacterial growth to 1, instantaneous complete killing); and the MV50 calculates the theoretical $\mathrm{MOI}$ at which a phage achieves a VP of 0.5 (half the theoretical maximum virulence).

\section{Data Visualisation}

Resulting graphs were visualized in R (v3.6.1) implemented through RStudio (v1.1.456) ${ }^{48}$ using the ggplot2 (v3.3.2) package ${ }^{49}$, with a custom colour-blind colour palette generated from ColorBrewer (https://colorbrewer2.org). 


\section{Results}

Sequence Similarity to Known Phages

The 30 Klebsiella phages were purified by multiple rounds of plating and genome sequencing showed a genome size range from $16,548 \mathrm{bp}$ to $268,500 \mathrm{bp}$. The Klebsiella phage genomes represented nine diverse, distinct genera, as determined by VIPtree (figure 1) and vConTACT2 (figure 2). Genome similarities between our phage isolates and known phages were determined using a combination of VIPtree and BLAST (table 3). Phage isolates were grouped based on genus-level similarity into groups A-I, referred to by their genera or closest level of identifiable taxonomy: Nonagvirus, unclassified family/genus, Tempevirinae unclassified, Myoviridae unclassified, Drulisvirus, Sugarlandvirus, Taipeivirus, Slopekvirus and Jiaodavirus. The phage isolates of groups B, C and D displayed lower sequence similarities to previously identified phages, hence they were not classified into known genera. The sequence data was deposited in the ENA, the associated project accession numbers are given in table 2 .

Alignments constructed in VIPtree ${ }^{33}$ showed varying levels of amino acid sequence identity but high gene synteny between our isolates and known phages (figures S3-10). The phage groups with the highest levels of amino acid identity and gene synteny to known phages were $F, G, H$ and I corresponding to Drulisvirus, Sugarlandvirus, Taipeivirus and Jiaodavirus genera respectively (fig. S811). Phage isolates in group $F$, Sugarlandvirus, showed the greatest similarity to previously described phages (fig. S8). All Sugarlandvirus isolates grouped with previously described vB_Kpn_IME260 and Klebsiella phage Sugarland, except vB_KaS-Veronica which represents a new distinct species based on ANI. The Sugarlandvirus isolates did exhibit variation in their tail fibre genes (figure S9; at $\sim 75 \mathrm{~kb}$ ). In contrast, phage isolates in group A, corresponding to the known genus of Nonagvirus, were less similar to previously known phages (fig. S4).

There was low sequence identity, but high gene synteny between our isolates and previously sequenced phages for groups B, C and D (fig. S5-7), a result of the isolates in these groups have unresolved taxonomies. Interestingly, analysis of the phage with the smallest genome, vB_KppS-Ant of group B, revealed $>99 \%$ nucleotide identity to region of the K. pneumoniae 30104 genome (data not shown).

For further similarity analysis between our isolates and known phages, the protein sequences of three marker genes (DNA polymerase, major capsid protein and terminase large subunit) were used to draw phylogenetic trees (fig. S12-20). Conserved branching patterns, indicating close evolutionary history were observed between most of our phage isolates and known phages, confirming that our isolates from groups $A, E, F, G, H$ and I belong to known genera (fig. S12,16-20), while groups $B, C$ and $D$ (fig. S13-15) do not. Given more distant relationships between the marker protein sequences, it is proposed that group $\mathrm{C}$ represent a novel genus of the subfamily Tempevirinae (fig. S14) and group D represent a novel genus of the Myoviridae family (fig. S15), while the marker genes of the phage isolate of group B had an even more distant relationship with phages of both Siphoviridae and Myoviridae families (fig. S13). Given the inovirus morphology observed for group B (fig. S2, black box), classification at the family level also remains unresolved.

\section{Host Range Testing}

Most phages had a host range which extended past their original isolation host and was not explained by depolymerase activity. The number of strains infected by each phage is displayed in figure 3 , further information on host range including strain and capsule type specificity is found in figure S1. Two of the isolated phage genera showed activity against only their isolation host, these are both comprised of putative temperate phages; group A (Nonagvirus; except KppS-Raw, which infected one additional strain) and group $B$ (unclassified family/genera). The lytic phage groups $D, E$ and $G$ (Myoviridae unclassified, Drulisvirus and Taipeivirus, respectively) showed lytic activity against 3-7 
Klebsiella strains and formed plaques in 1-7 of those strains. While group $\mathrm{F}$ (Sugarlandvirus) phages had activity against 16 Klebsiella strains and plaques on 10 strains. Group C (Tempevirinae unclassified) was highly variable, with KaS-Gatomon and KaS-Ahsoka forming plaques on only their isolation strain, while Kpp-Samwise formed plaques in seven strains and activity against a further five strains. The broadest range were lytic phages belonging to the subfamily Tevenvirinae; assigned to the groups H (Slopekvirus) and I (Jiaodavirus), demonstrated activity against 23 and 17 strains respectively and were able to produce plaques on 13 and 9 strains respectively. Within the genera host range varied between phages; from the group $\mathrm{H}$ (Slopekvirus), the phage KoM-Pickle was only able to form plaques on its isolation host, yet showed some level of activity against 22 out of 23 other Klebsiella spp. (figure 3; figure S1).

\section{Phage Annotation}

Genome annotation is notoriously difficult with phages, given the extremes of sequence variation evident in all phage proteins ${ }^{50,51}$. As a first indication for comparisons, PROKKA was used and identified key phage genes e.g. portal proteins, capsid genes, tail proteins, components of the DNA replication and packaging machinery. Additionally, $\mathrm{PhoH}$ was a common feature in 18 of the 30 phages sequenced, including phages from groups D, F, G and $\mathrm{H}$ (Myoviridae unclassified, Sugarlandvirus, Taipeivirus and Slopekvirus, respectively). Holin and lysin pairs were identified in groups A, C and G (Nonagvirus, Tempevirinae unclassified and Taipeivirus, respectively), while endolysin and Rz1 spanin complex genes were identified in groups E and I (Drulisvirus and Jiaodavirus, respectively).

Most of our isolated phages encode at least one gene annotated as a putative "tail-fibre" or "tail-spike" protein (table S2). Intial structural predictions suggested that these proteins adopt beta-helical structures, a common protein architecture of both tail-spike proteins and the capsule depolymerase enzymes that are suggested to have evolved from these purely structural proteins ${ }^{52-54}$. These proteins also contained predicted enzymatic domains, such as the Pectate_lyase_3 domain or Peptidase_S74 domain, which have been identified in other phage encoded depolymerases ${ }^{55-58}$. Several of the analysed phage groups: B, C, F, H and I, did not have a predicted tail-fiber depolymerase protein. It is unclear whether these phages genuinely lack the activity, or that detection was hampered by extremes of sequence variation ${ }^{50,51}$.

For those phages where a candidate depolymerase could be identifed, protein sequence relationships were mapped on a tree (figure 4). Phages in the group A (Nonagvirus), and group E (Drulisvirus) encode a similar type of putative predicted depolymerase protein. The putative depolymerases from Drulisvirus phages share high sequence conservation ( 95\% identity, $100 \%$ query) to the experimentally characterised depolymerase Kpv74_56 from the closely related Drulisvirus, Klebsiella pneumoniae phage $\mathrm{KpV74}{ }^{59}$, and both sets of phage infect K2 capsule-producing strains of Klebsiella (fig. 4). Interestingly, the phages of two further genera; group D (Myoviridae unclassified; vB_KqMEowyn) and group G (Taipeivirus; vB_KqvM-LilBean, vB_KqvM-Bilbo and vB_KqvM-Westerburg) each contain multiple putative depolymerase-like proteins (7, 4, 4, 5 respectively; fig. 4). The putative depolymerase-like proteins of vB_KqM-Eowyn (D, Myoviridae unclassified) show low identity to previously characterised depolymerases (fig. 4). From our host range assays these phages showed activity towards strains that produced a small subset of K-antigens (vB_KqM-Eowyn - KL16, KL110; vB_KqvM-LilBean - KL35; vB_KqvM-Bilbo - KL35, K2 and vB_KqvM-Westerburg - KL35, KL2, KL3).

\section{Morphology of Phages}

Phage-induced plaques in the lawns of host bacteria varied in size, and also varied in the presence or absence of halos surrounding the phage plaques. Representative images of phage plaques for each described genera are presented in figure 5 and images for all phages are included in supplementary figure S2. A diffuse halo around the phage plaques was observed in 26 of the 30 phage isolates (table S3 and fig. S2). 
Representative TEM images of the described phage genera are provided in figure 5. Average tail length and capsid widths are given in supplementary table S3 based on 30 phage particles, a representative TEM image for each genera is displayed in figure S2. Of the phages imaged, 13 are myovirus, 13 are siphovirus, three are podovirus and one inovirus (fig. S2). The largest phage in this study was KvM-Eowyn of group D (Myoviridae unclassified), which had a capsid width of $140 \mathrm{~nm}$ and tail length of $140 \mathrm{~nm}$, this corresponded to the largest genome at $269 \mathrm{Kbp}$. The smallest phage was $\mathrm{KqP}-G o l i a t h$, a podovirus of group E (Drulisvirus), with a capsid width of $41 \mathrm{~nm}$ and tail length of 10 $\mathrm{nm}$, had the second smallest genome at $44 \mathrm{Kbp}$, after the filamentous prophage KppS-Ant, group $B$ (unclassified family/genus). KppS-Raw, a group A (Nonagvirus) siphovirus, had a comparable capsid size of $46 \mathrm{~nm}$, but a substantially longer tail $(153 \mathrm{~nm})$ and larger genome of $61 \mathrm{Kbp}$. The smallest icosahedral phage virions, group $\mathrm{E}$ (Drulisvirus), produced the largest plaques and halos, hence the name KqP-Goliath (table S1, figure S2).

\section{Phage Lysis period and Virulence in Host Strains}

The lysis period and virulence indices (VP and MV50) of each phage, in their relevant isolation host strain in LB at $37^{\circ} \mathrm{C}$, are displayed in Figure 6. For six phages a lysis period was not achieved (KppSEggy, KppS-Pokey, KppS-Ant, KpM-Milk, KpM-KalD and KpM-SoFaint), the growth curve of the host bacteria was dampened (except KppS-Ant), but the culture density did not crash compared to the positive control - indicative of temperate phages. These phages also demonstrated a below average VP, close to 0 (with the exception of KpM-KalD), indicating little difference in the area under the curve between the control and phage infected cultures (fig. 6). Of the remaining phages that did exhibit a lysis period, the median time was $\sim 70$ minutes, ranging from 15 minutes to 210 minutes. There was no correlation between lysis period and the virulence measures (figure 6 ; statistical data not shown), implying that lysis period was not the driving factor. The average VP for the phages was 0.33 (range $0.06-0.64$ ) and MV50 was achieved with a projected MOI of $4.17 \times 10^{17}$ (range $3.5 \times 10^{-7}-1 \times 10^{19}$ ), when the putative temperate phages from groups $A$ and $B$ were excluded. The putative temperate phages had lower virulence measures, reinforcing previous findings. For these, the average VP was 0.06 (range $-0.07-0.36$ ) and for the MV50 an MOI of $6.00 \times 10^{58}$ (range $0.012-3 \times 10^{59}$ ). Phages from groups C, E, F and I (Tempevirinae unclassified, Drulisvirus, Sugarlandvirus, Jiaodavirus, respectively) had the highest virulence (VP) (range 0.12-0.64, average, 0.42) and below average MV50 (range 3.5×10 ${ }^{7}-1.30 \times 10^{5}$, average $9.30 \times 10^{3}$ ) indicated that they killed their hosts quickest, and needed a lower phage:host ratio to achieve this. It is however difficult to generalise for each genus because the two metrics varied between phages of the same genera. The virulence index results are specific to each phage and conditions assessed, and therefore are not directly comparable between phages grown on different hosts. 


\section{Discussion}

Klebsiella-infecting phages, belonging to nine phylogenetically distinct lineages, were isolated from water samples sourced from different environments. The 30 independent phages were discovered using a panel of Klebsiella species - both clinical isolates and environmental strains - belonging to five bacterial species: Klebsiella pnuemoniae, $K$. oxytoca, $K$. quasipneumoniae, $K$. aerogenes, and $K$. variicola. The Klebsiella spp. used span 18 different capsule types.

This study discovered several phages and phage genera that have not previously been described. Phylogenetic analysis revealed that the filamentous phage vB_KppS-Ant of group B did not significantly cluster with any known phages at the shared protein-level, and therefore represents a novel genus. The genome reconstruction of this ssDNA phage is unexpected; our sequencing strategy was optimised for dsDNA, however previously studies have demonstrated Illumina sequencing to be inefficient yet successful at sequencing ssDNA phages ${ }^{60}$. The high sequence similarity of phage vB_KppS-Ant to a region of the K. pneumoniae 30104 genome (>99 \%) indicates that it is an induced prophage, which represents a new genus. By combining shared protein network analysis and marker gene phylogenetic tree analysis, we identified two further novel phage genera: group C (Tempevirinae unclassified) and group D (Myoviridae unclassified).

\section{Factors determining host range}

In general, comparative genomics revealed sequence conservation over a large portion of their genomes, with variability in only a few genes (fig. S4-11), with divergent characteristics in terms of host range and virulence. Despite several of these phage species having been identified in previous studies, there are examples where these showed differences in host-range. Thus, even within the small sample presented here, there is information to be gained about the factors determining hostrange.

It has been suggested that selection pressure imposed from the use of a host is sufficient to amplify nongenetic variants of a phage that can cross host-range ${ }^{47,61}$, and it also remains possible that uncharacterised genes, encoding proteins of unknown function, could adapt a given phage to a distinct host ${ }^{62}$. By way of example, the phages of group $F$ (Sugarlandvirus) showed a high degree of similarity between genome sequences, with most variation concentrated in their tail fibre genes. Tail fibres mediate interaction with host cell receptors and are frequently rearranged in phages, allowing them to adhere to bacterial hosts ${ }^{63}$. These tail fibres can include domains with enzymatic function, enabling degradation of host-specific features such as polysaccharide capsules ${ }^{64}$. Three phages with $>99 \%$ similarity in genome sequence were isolated on distinct hosts: K. pneumoniae 30104 (vB_KppS_Ponyo and vB_KppS_Totoro) or K. aerogenes 30053 (vB_KaS-Benoit). vB_KppS_Ponyo and vB_KaS-Benoit are $100 \%$ identical at the nucleotide level, and vB_KppS_Totoro has a single SNP. Further host range analysis showed that the two phages propagated on K. pneumoniae 30104 had comparable host ranges, while the phage propagated on $K$. aerogenes 30053 had a different host range (fig. S1). This indicates that the propagation host, influenced host range, and that genome sequence cannot be used to infer host-range without careful consideration ${ }^{65}$. This is a vitally important point to consider when choosing phages for phage therapy, we cannot generalise based on genome similarity, when two phages of the same species and even those with $100 \%$ identity do not necessarily behave in the same way, particularly depending on prevailing conditions ${ }^{66}$.

While multiple features of strain-specific bacterial immunity can protect against phage replication in a given lineage, in Klebsiella the primary defense against both phages and antibiotics is a protective polysaccharide capsule ${ }^{67,68}$. This capsule forms the outermost layer of the Klebsiella cell and acts as an important virulence factor ${ }^{69}$. There are currently at least 77 different serologically defined Klebsiella capsule types ${ }^{70,71}$. Currently the use of whole genome methods for capsule typing is favoured ${ }^{32}$ and showed that our Klebsiella panel encompassed 18 capsule types, including three KL2 
strains, an important capsule type in clinical infections and therefore of interest to develop effective therapies against ${ }^{72}$.

Klebsiella phages have repeatedly shown to be specific to host capsule types ${ }^{73-75}$, this is often linked to phage sugar-degrading enzymes called depolymerases that target specific capsule types ${ }^{56,59,76}$. Given the broad host ranges observed in our collection of phages (fig. 3) and depolymerase indicative halos ${ }^{77}$ in $83 \%$ of our phage isolates categorised as groups C-I (table S3), we sought to identify depolymerases genes. A surprisingly high number of putative depolymerase genes (7) were identified in the group $\mathrm{D}$ (Myoviridae unclassified) phage KvM-Eowyn, which only produced plaques in its KL16 host strain, but showed potential depolymerase activity against a KL110 producing strain. Klebsiella phages encoding up to 11 different polysaccharide depolymerase genes have previously been characterised, but have also been shown to infect a correspondingly wide range of Klebsiella capsule types ${ }^{78}$, indicating the need to expand our Klebsiella panel. The phages in group G (Taipeivirus) encoded 4-6 each depolymerases, these phages produced plaques against the clinically relevant KL2 capsule type. The phages of group A (Nonagvirus) and group $\mathrm{E}$ (Drulisvirus) had only one depolymerase gene each, in line the with the average number of expected depolymerases ${ }^{53}$. Characterisation of these putative depolymerase genes is important to further investigate the potential extended host range of these phages, beyond the Klebsiella strains included in this analysis. Conversely, genes encoding depolymerases were not identifiable in all of our halo producing phages, including in some of the broadest ranging phages of groups F, H and I (Sugarlandvirus, Slopekvirus and Jiaodavirus). The phage with the broadest host-range, KoM-MeTiny of group $\mathrm{H}$ (Slopekvirus), produced halos, showed depolymerase/lysis activity against $79 \%$ of the Klebsiella strains, and produced plaques on $42 \%$ of the Klebsiella tested, which included nine different capsule types, but had no identifiable depolymerase genes. We suggest that this is either because conventional depolymerases are not essential for the phages to permeate the capsule layer, or because of shortcomings in the sequence-based annotation of phage genomes ${ }^{50,51}$. There is limited sequence conservation between many of the putative tail-fiber/tail-spike depolymerase proteins from our collection of phages to those that have been biochemically validated, therefore further characterisation of these proteins will be critical for future optimisation of phage cocktails for therapeutic uses.

Application to future phage-based therapy

As expected with lytic phages, the phages isolated caused crashes in the Klebsiella cultures, but no phage was able to suppress Klebsiella growth for more than 12 hours (Figure S3). Phage cocktails are frequently used to improve the impact of phages on a Klebsiella population and are currently considered crucial for the efficacy of phage therapy ${ }^{79-81}$. Phage cocktails benefit from complementarity and redundancy between the combined phages to overcome host-evolved phageresistance ${ }^{82}$, which may account for the resurgences seen in our Klebsiella cultures. After phage selection, the testing of phage combinations is imperative to ensure no adverse effects occur, such antagonistic phage interactions, which could result in bacterial stress responses or biofilm formation, as seen with sub-lethal antibiotic use ${ }^{83,84}$. The phages described in this study have been supplied for use in compassionate phage therapy, requiring rigorous and lengthy testing to identify which phages were active against the clinically infective Klebsiella and to ensure safety.

For use in phage therapy, phages must not encode toxins or AMR genes ${ }^{79,81,85}$. None of our Klebsiella phages contained identifiable toxins or AMR genes. Other factors may be considerations for taking phages such as these into preclinical trials. For example, the host infection dynamics and low virulence of the phages of group A (Nonagvirus) indicate that these isolates may also be temperate and/or may not be curative on infections. The temperate phage KppS-Ant (group B), as the only phage with an identifiable integrase for lysogeny would be excluded for phage therapy purposes ${ }^{86}$. Additionally, the host infection dynamics and low virulence of the phages of group A (Nonagvirus) indicates a temperate lifestyle, paired with the knowledge that the closest related known phages are 
temperate, thus excluding these isolates for phage therapy. Group I (Jiaodavirus) phages encode a Hoc-like protein, which in phage T4 has been demonstrated to be highly immunogenic ${ }^{87}$. It should be established if these phages cause an immune response before using them for phage therapy.

The genomic information and experimental data presented here for phage groups $\mathrm{C}, \mathrm{F}, \mathrm{G}$ and $\mathrm{H}$ (Tempevirinae unclassified, Sugarlandvirus, Taipeivirus and Slopekvirus, respectively) indicates that they are lytic, not lysogenic, and thus suitable for preclinical evaluation. We suggest that the isolates of phage group $\mathrm{F}$ (Sugarlandvirus) and group $\mathrm{H}$ (Slopekvirus) are the best candidates for future development in phage therapy, given their broad host range, high virulence, short latency period and lack of potentially harmful genes. Taken together our data suggests that in order to provide universal, effective phage therapy against Klebsiella infections, a phage cocktail comprised of multiple diverse phages should be developed. This cocktail would prevent growth of Klebsiella cells resistant to a single phage or phage groups we isolated.

\section{Conclusions}

A diverse range of Klebsiella phages were isolated from various environmental samples. The temperate phages discovered in this work may have interesting future applications, but are unsuitable for clinical applications. Phage isolates belonging to the Sugarlandvirus and Slopekvirus genera were deemed most suitable for phage therapy, due to their broad host range, high virulence, short lysis period and lack of potentially harmful genes. Despite some of our phage isolates grouping into a single previously described phage species, within species variation in both host range and virulence were observed. This demonstrates the necessity to microbiologically characterise phages before selecting candidates for therapeutic use.

\section{Acknowledgments}

We acknowledge the help of Severn Trent for enabling us to collect sewage samples from Spernal sewage treatment works. We acknowledge the Midlands Regional Cryo-EM Facility, hosted at the Warwick Advanced Bioimaging Research Technology Platform, for use of the JEOL 2100Plus, supported by MRC award reference MC_PC_17136. Genome sequencing was provided by MicrobesNG (http://www.microbesng.uk). This work was supported by a Warwick Integrative Synthetic Biology (WISB) early career fellowship, funded jointly by BBSRC and EPSRC to EJ and the Monash Warwick Alliance Accelerator Fund October 2019 to EJ and TL. Bioinformatics analysis was carried out by infrastructure provided by MRC CLIMB (MR/T030062/1). The work has also been supported by PhD fellowships awarded to LK, LG, GM and HS funded by BBSRC/ESPRC DTPS. 
Figure 1. Protein level phylogenetic tree, generated by VIPtree. Klebsiella phage isolates (回). A-I denote phage groups with the following genera: A. Nonagvirus; B. unclassified family/genus; C. Tempevirinae unclassified; D. Myoviridae unclassified; E. Drulisvirus; F. Sugarlandvirus; G. Taipeivirus; H. Slopekvirus and I. Jiaodavirus. Icons indicate phage morphology.

Figure 2. Network analysis of phage-encoded proteins calculated with vConTACT2. Coloured, numbered nodes represent our Klebsiella phage isolates, coloured according to the phage group and subsequent genera to which each phage belongs. Numbers within nodes indicate the lab identification numbers (see table 2). Smaller, black nodes represent previously sequenced phages as references. Edges between nodes represent shared proteins, such that many connecting edges implies greater pairwise shared protein content. Phage nodes are clustered based on shared proteins, with a springembedded (force directed) layout visualisation created in Cytoscape.

Figure 3. Number of Klebsiella strains infected by each bacteriophage out of a possible 24. Plaques, indicates the number of strains a phage replicated in and resulted in plaques on overlay agar; Clearance, indicates the additional number of strains the phage cleared or partially cleared the bacterial lawn during spot testing analysis (Figure S1). Totals include the isolation host. Bars marked with \# denote an incomplete data set. Coloured outlines relate to the phage groups, as identified in figure 1 , with genera annotated for each group.

Figure 4. Phylogenetic tree of previously biochemically characterised depolymerase proteins that target Klebsiella spp. and putative depolymerase proteins in our phage isolates. Sequences were aligned with Muscle using SeaView. Phylogenetic tree construction was performed with MegaX with 500 bootstrap calculations using the LG model. Tree topology searches were performed using a combination of $\mathrm{NNI}$ and $\mathrm{NJ} / \mathrm{BioNJ}$. The tree was subsequently visualised and annotated using iTOL (v4). Depolymerases highlighted in colour blocks have overlapping potential target $\mathrm{K}$ antigens.

Figure 5. Phage morphology, showing representative TEM and plaque images for each phage group. TEM and plaque assays were performed as described in the methods. Scale bars on the TEM images represent $200 \mathrm{~nm}$. Further images of all phages can be found in the supplementary materials, figure S2.

Figure 6. Lysis period and virulence indices are not correlated, but temperate phages are less virulent. Panel (A) displays the virulence index of the phages. Virulence index is a quantified measure of the phage, in their respective isolation host (table 2), in LB with $5 \mathrm{mM}$ each of $\mathrm{CaCl}_{2}$ and $\mathrm{MgCl}_{2}$, at $37 \mathrm{C}$. Panel (B) displays the MV50, the MOI at which each phage achieves $50 \%$ of their maximal theoretical virulence. Both of these virulence measures are described in more detail by Storms ${ }^{47}$. Panel $(C)$ displays the lysis period of the phage. Where this is left blank, a lysis period could not be established usually indicating temperate lifestyle. Coloured outlines relate to the phage groups, as identified in figure 1, with genera annotated for each group. Dashed lines indicate the median values for each metric.

Table 1. Details of Klebsiella species and strains used in this study. Capsule types are given where applicable, alongside the origin of the strain and indication of use as an isolation host.

Table 2. Phage isolate details. Lab ID refers to the laboratory identification number, source of isolation, indicates where the water sample was collected for phage enrichment and isolation and the strain of isolation indicates Klebsiella sp. strain on which 3 rounds of plaque assay isolation were performed. Accession numbers refers to associated project accession numbers assigned by the ENA for each phage.

Table 3. Phage taxonomy and similarity to closest sequenced phage. Family, Subfamily and Genus are assigned based on the clustering patterns observed in vConTACT2 analysis (fig. 2), and conserved branching patterns observed in the marker gene phylogenetic trees (fig. S12-20). ANI refers to Average Nucleotide Identity calculated with orthoANI (https://www.ezbiocloud.net/tools/ani). Details for "comparison phage" relate to details of previously sequenced phages available in public databases used for ANI analysis. 
Figure S1. Phage host range matrix. Host range was determined by spot testing on LB agar overlay plates against the Klebsiella spp. in our panel. Dark blue indicates host of isolation, in which plaques were produced, Light blue none-host strains where plaques were produced, Red indicates that spot testing caused the lawn to clear, but no plaques were visible, Dark red indicates some incomplete reduction was observed in the bacterial lawn, but no plaques and Grey indicates no observed effect.

Figure S2. Phage morphology; TEM and plaque morphology. The clearest TEM images were selected for each phage, scales vary for each image and $\mathrm{nm}$ scale bars are included for each image. An image of the plaques produced by each phage is included following overnight incubation at $37^{\circ} \mathrm{C}$ on agar overlay plates.

Figure S3. Impact of each phage isolate on the growth curves of its reciprocal isolation host, grown in LB at $37^{\circ} \mathrm{C}$.

Figure S4. Group A (Nonagvirus) amino acid alignment of our phage isolates and reference genomes identified in vConTACT2 analysis, drawn in VIPtree.

Figure S5. Group B (unclassified family/genus) amino acid alignment of our phage isolates and reference genomes identified in vConTACT2 analysis, drawn in VIPtree.

Figure S6. Group C (Tempevirinae unclassified) amino acid alignment of our phage isolates and reference genomes identified in vConTACT2 analysis, drawn in VIPtree.

Figure S7. Group D (Myoviridae unclassified) amino acid alignment of our phage isolates and reference genomes identified in vConTACT2 analysis, drawn in VIPtree.

Figure S8. Group E (Drulisvirus) amino acid alignment of our phage isolates and reference genomes identified in vConTACT2 analysis, drawn in VIPtree.

Figure S9. Group F (Sugarlandvirus) amino acid alignment of our phage isolates and reference genomes identified in vConTACT2 analysis, drawn in VIPtree.

Figure S10. Group G (Taipeivirus) amino acid alignment of our phage isolates and reference genomes identified in vConTACT2 analysis, drawn in VIPtree.

Figure S11. Combined group H (Slopekvirus) and group I (Jiaodavirus) amino acid alignment of our phage isolates and reference genomes identified in vConTACT2 analysis, drawn in VIPtree.

Figure S12. Phylogenetic trees of DNA polymerase and terminase large subunit of phage Group A (Nonagvirus). Drawn with RaxML using the GAMMA model of heterogeneity and the maximumlikelihood method based on the JTT substitution matrix. Trees contain phage isolates from group A and reference phages identified by vConTACT2 and closest BLASTP hits. Coloured bar indicates known genera of reference phages.

Figure S13. Phylogenetic trees of terminase large subunit and major capsid protein of phage Group B (unclassified family/genus). Drawn with RaxML using the GAMMA model of heterogeneity and the maximum-likelihood method based on the JTT substitution matrix. Trees contain phage isolates from group B and reference phages identified by vConTACT2 and closest BLASTP hits. Coloured bar indicates known genera of reference phages.

Figure S14. Phylogenetic tree of terminase large subunit of phage Group C (Tempevirinae unclassified). Drawn with RaxML using the GAMMA model of heterogeneity and the maximumlikelihood method based on the JTT substitution matrix. Tree contains phage isolates from group $C$ and reference phages identified by vConTACT2 and closest BLASTP hits. Coloured bar indicates known genera of reference phages.

Figure S15. Phylogenetic trees of DNA polymerase, major capsid protein and terminase large subunit of phage Group D (Myoviridae unclassified). Drawn with RaxML using the GAMMA model of 
heterogeneity and the maximum-likelihood method based on the JTT substitution matrix. Trees contain phage isolates from group D and reference phages identified by vConTACT2 and closest BLASTP hits. Coloured bar indicates known genera of reference phages.

Figure S16. Phylogenetic tree of DNA polymerase of phage Group E (Drulisvirus). Drawn with RaxML using the GAMMA model of heterogeneity and the maximum-likelihood method based on the JTT substitution matrix. Tree contains phage isolates from group $E$ and reference phages identified by vConTACT2 and closest BLASTP hits. Coloured bar indicates known genera of reference phages.

Figure S17. Phylogenetic trees of DNA polymerase, major capsid protein and terminase large subunit of phage Group F (Sugarlandvirus). Drawn with RaxML using the GAMMA model of heterogeneity and the maximum-likelihood method based on the JTT substitution matrix. Trees contain phage isolates from group $\mathrm{F}$ and reference phages identified by vConTACT2 and closest BLASTP hits. Coloured bar indicates known genera of reference phages.

Figure S18. Phylogenetic trees of DNA polymerase, major capsid protein and terminase large subunit of phage Group G (Taipeivirus). Drawn with RaxML using the GAMMA model of heterogeneity and the maximum-likelihood method based on the JTT substitution matrix. Trees contain phage isolates from group $\mathrm{G}$ and reference phages identified by vConTACT2 and closest BLASTP hits. Coloured bar indicates known genera of reference phages.

Figure S19. Phylogenetic trees of DNA polymerase, major capsid protein and terminase large subunit of phage Group H (Slopekvirus). Drawn with RaxML using the GAMMA model of heterogeneity and the maximum-likelihood method based on the JTT substitution matrix. Trees contain phage isolates from group $\mathrm{H}$ and reference phages identified by vConTACT2 and closest BLASTP hits. Coloured bar indicates known genera of reference phages.

Figure S20. Phylogenetic trees of DNA polymerase, major capsid protein and terminase large subunit of phage Group I (Jiaodavirus). Drawn with RaxML using the GAMMA model of heterogeneity and the maximum-likelihood method based on the JTT substitution matrix. Trees contain phage isolates from group I and reference phages identified by vConTACT2 and closest BLASTP hits. Coloured bar indicates known genera of reference phages.

Table S1. List of characterised Klebsiella targeting depolymerase tail-fibre proteins used to construct the phylogenetic tree (Figure 4).

Table S2. List of putative depolymerase tail-fibre proteins from novel phages characterised in this study. Each sequence was analysed by BLASTP, HMMer and HHpred using the default parameters. The top HHpred hits are described for each protein. These sequences in combination with the sequences of characterised depolymerases from phages described in Table S1 were used to construct the phylogenetic tree (Figure 4).

Table S3. Phage measurements; phage particle measurement calculated from TEM imaging and measured with imageJ, and genome size in nucleotide base pairs. 


\section{References:}

1. Podschun R, Ullmann U. Klebsiella spp. as nosocomial pathogens: epidemiology, taxonomy, typing methods, and pathogenicity factors. Clinical microbiology reviews 1998;11:589-603.

2. Dhesi Z, Enne VI, Brealey D et al. Organisms causing secondary pneumonias in COVID-19 patients at 5 UK ICUs as detected with the FilmArray test. medRxiv 2020:2020.2006.2022.20131573.

3. He Y, Li W, Wang Z et al. Nosocomial infection among patients with COVID-19: A retrospective data analysis of 918 cases from a single center in Wuhan, China. Infection Control \& Hospital Epidemiology 2020:1-2.

4. Zhu X, Ge Y, Wu T et al. Co-infection with respiratory pathogens among COVID-2019 cases. Virus Research 2020;285:198005.

5. Jameson E, Quareshy M, Chen Y. Methodological considerations for the identification of choline and carnitine-degrading bacteria in the gut. Methods 2018;149:4248.

6. Yan Q, Gu Y, Li X et al. Alterations of the Gut Microbiome in Hypertension. Front Cell Infect Microbiol 2017;7:381.

7. Atarashi K, Suda W, Luo C et al. Ectopic colonization of oral bacteria in the intestine drives TH1 cell induction and inflammation. Science 2017;358:359-365.

8. Singh L, Cariappa M, Kaur M. Klebsiella oxytoca: An emerging pathogen? Medical Journal Armed Forces India 2016;72:S59-S61.

9. Rodríguez-Medina N, Barrios-Camacho $\mathrm{H}$, Duran-Bedolla J et al. Klebsiella variicola: an emerging pathogen in humans. Emerging microbes \& infections 2019;8:973-988.

10. Shankar C, Nabarro LE, Anandan S et al. Extremely High Mortality Rates in Patients with Carbapenem-resistant, Hypermucoviscous Klebsiella pneumoniae Blood Stream Infections. J Assoc Physicians India 2018;66:13-16.

11. Tacconelli $\mathrm{E}$, Magrini $\mathrm{N}$, Kahlmeter $\mathrm{G}$ et al. Global priority list of antibiotic-resistant bacteria to guide research, discovery, and development of new antibiotics. World Health Organization 2017;27.

12. Navon-Venezia S, Kondratyeva K, Carattoli A. Klebsiella pneumoniae: a major world wide source and shuttle for antibiotic resistance. FEMS Microbiol Rev 2017;41:252275.

13. Sanchez GV, Master RN, Clark RB et al. Klebsiella pneumoniae antimicrobial drug resistance, United States, 1998-2010. Emerging infectious diseases 2013;19:133.

14. Elemam A, Rahimian J, Mandell W. Infection with panresistant Klebsiella pneumoniae: a report of 2 cases and a brief review of the literature. Clinical infectious diseases 2009;49:271-274.

15. Sonnevend Á, Ghazawi A, Hashmey R et al. Multihospital occurrence of pan-resistant Klebsiella pneumoniae sequence type 147 with an ISEcp1-directed blaOXA-181 insertion in the mgrB gene in the United Arab Emirates. Antimicrobial agents and chemotherapy 2017;61.

16. Turner P, Ashley EA, Celhay OJ et al. ACORN (A Clinically-Oriented Antimicrobial Resistance Surveillance Network): a pilot protocol for case based antimicrobial resistance surveillance. Wellcome Open Research 2020;5.

17. ECDC ECfDPaC. Surveillance Atlas of Infectious Diseases. 2019. 
18. Patro LPP, Rathinavelan T. Targeting the sugary armor of Klebsiella species. Frontiers in Cellular and Infection Microbiology 2019;9.

19. Mollers M, Lutgens SP, Schoffelen AF et al. Cost of Nosocomial Outbreak Caused by NDM-1-Containing Klebsiella pneumoniae in the Netherlands, October 2015-January 2016. Emerg Infect Dis 2017;23:1574-1576.

20. Ben-David D, Kordevani R, Keller $\mathrm{N}$ et al. Outcome of carbapenem resistant Klebsiella pneumoniae bloodstream infections. Clinical Microbiology and Infection 2012;18:54-60.

21. El Haddad L, Harb CP, Gebara MA et al. A systematic and critical review of bacteriophage therapy against multidrug-resistant ESKAPE organisms in humans. Clinical Infectious Diseases 2019;69:167-178.

22. Dedrick RM, Guerrero-Bustamante CA, Garlena RA et al. Engineered bacteriophages for treatment of a patient with a disseminated drug-resistant Mycobacterium abscessus. Nat Med 2019;25:730-733.

23. Schooley RT, Biswas B, Gill JJ et al. Development and use of personalized bacteriophage-based therapeutic cocktails to treat a patient with a disseminated resistant Acinetobacter baumannii infection. Antimicrobial agents and chemotherapy 2017;61:e00954-00917.

24. Edwards RA, Rohwer F. Opinion: viral metagenomics. Nature reviews Microbiology 2005;3:504.

25. Brussow H, Canchaya $\mathrm{C}$, Hardt WD. Phages and the evolution of bacterial pathogens: from genomic rearrangements to lysogenic conversion. Microbiol Mol Biol Rev 2004;68:560602 , table of contents.

26. Adriaenssens $E M$, Sullivan $M B$, Knezevic $P$ et al. Taxonomy of prokaryotic viruses: 2018-2019 update from the ICTV Bacterial and Archaeal Viruses Subcommittee. Archives of virology 2020:1-8.

27. Sambrook J, Fritsch EF, Maniatis T. Molecular cloning: a laboratory manual. (Cold spring harbor laboratory press). 1989.

28. Bolger AM, Lohse M, Usadel B. Trimmomatic: a flexible trimmer for Illumina sequence data. Bioinformatics 2014;30:2114-2120.

29. Bankevich A, Nurk S, Antipov D et al. SPAdes: a new genome assembly algorithm and its applications to single-cell sequencing. J Comput Biol 2012;19:455-477.

30. Seemann T. Prokka: rapid prokaryotic genome annotation. Bioinformatics 2014;30:2068-2069.

31. Michniewski S, Redgwell T, Grigonyte A et al. Riding the wave of genomics to investigate aquatic coliphage diversity and activity. Environ Microbiol 2019;21:2112-2128.

32. Wick RR, Heinz E, Holt KE et al. Kaptive Web: User-Friendly Capsule and Lipopolysaccharide Serotype Prediction for Klebsiella Genomes. J Clin Microbiol 2018;56:JCM.00197-00118.

33. Rohwer F, Edwards R. The Phage Proteomic Tree: a genome-based taxonomy for phage. J Bacteriol 2002;184:4529-4535.

34. Lee I, Kim YO, Park S-C et al. OrthoANI: an improved algorithm and software for calculating average nucleotide identity. International journal of systematic and evolutionary microbiology 2016;66:1100-1103.

35. Adriaenssens E, Brister JR. How to name and classify your phage: an informal guide. Viruses 2017;9:70.

36. Bolduc B, Jang HB, Doulcier $\mathrm{G}$ et al. vConTACT: an iVirus tool to classify doublestranded DNA viruses that infect Archaea and Bacteria. PeerJ 2017;5:e3243. 
37. Shannon P, Markiel A, Ozier O et al. Cytoscape: a software environment for integrated models of biomolecular interaction networks. Genome research 2003;13:24982504.

38. Katoh K, Standley DM. MAFFT multiple sequence alignment software version 7: improvements in performance and usability. Molecular biology and evolution 2013;30:772780.

39. Stamatakis A. RAxML version 8: a tool for phylogenetic analysis and post-analysis of large phylogenies. Bioinformatics 2014;30:1312-1313.

40. Yu G, Smith DK, Zhu H et al. ggtree: an r package for visualization and annotation of phylogenetic trees with their covariates and other associated data. Methods in Ecology and Evolution 2017;8:28-36.

41. Yu G, Lam TT-Y, Zhu H et al. Two Methods for Mapping and Visualizing Associated Data on Phylogeny Using Ggtree. Molecular biology and evolution 2018;35:3041-3043.

42. Revell LJ. phytools: an R package for phylogenetic comparative biology (and other things). Methods in ecology and evolution 2012;3:217-223.

43. Edgar RC. MUSCLE: multiple sequence alignment with high accuracy and high throughput. Nucleic Acids Res 2004;32:1792-1797.

44. Gouy M, Guindon S, Gascuel O. SeaView version 4: a multiplatform graphical user interface for sequence alignment and phylogenetic tree building. Molecular biology and evolution 2010;27:221-224.

45. Kumar S, Stecher G, Li M et al. MEGA X: molecular evolutionary genetics analysis across computing platforms. Molecular biology and evolution 2018;35:1547-1549.

46. Letunic I, Bork P. Interactive Tree Of Life (iTOL) v4: recent updates and new developments. Nucleic acids research 2019;47:W256-W259.

47. Storms ZJ, Teel MR, Mercurio K et al. The Virulence Index: A Metric for Quantitative Analysis of Phage Virulence. Phage 2020;1:27-36.

48. Allaire J. RStudio: integrated development environment for R. Boston, MA 2012;770:394.

49. Wickham H. ggplot2: elegant graphics for data analysis. (springer). 2016.

50. McNair K, Aziz RK, Pusch GD et al. Phage genome annotation using the RAST pipeline. In: Bacteriophages. (Springer). 2018; pp. 231-238.

51. McNair K, Zhou C, Dinsdale EA et al. PHANOTATE: a novel approach to gene identification in phage genomes. Bioinformatics 2019;35:4537-4542.

52. Squeglia F, Maciejewska B, Łątka A et al. Structural and Functional Studies of a Klebsiella Phage Capsule Depolymerase Tailspike: Mechanistic Insights into Capsular Degradation. Structure 2020.

53. Latka A, Leiman PG, Drulis-Kawa Z et al. Modelling the architecture of depolymerasecontaining receptor binding proteins in Klebsiella phages. Frontiers in microbiology 2019;10:2649.

54. Latka A, Maciejewska B, Majkowska-Skrobek G et al. Bacteriophage-encoded virionassociated enzymes to overcome the carbohydrate barriers during the infection process. Applied Microbiology and Biotechnology 2017;101:3103-3119.

55. Lin T-L, Hsieh P-F, Huang Y-T et al. Isolation of a bacteriophage and its depolymerase specific for K1 capsule of Klebsiella pneumoniae: implication in typing and treatment. The Journal of infectious diseases 2014;210:1734-1744. 
56. Majkowska-Skrobek G, Łątka A, Berisio R et al. Capsule-targeting depolymerase, derived from Klebsiella KP36 phage, as a tool for the development of anti-virulent strategy. Viruses 2016;8:324.

57. Kimura K, Itoh Y. Characterization of poly- - -glutamate hydrolase encoded by a bacteriophage genome: possible role in phage infection of Bacillus subtilis encapsulated with poly- $\gamma$-glutamate. Applied and Environmental Microbiology 2003;69:2491-2497. 58. Pires DP, Oliveira H, Melo LD et al. Bacteriophage-encoded depolymerases: their diversity and biotechnological applications. Applied microbiology and biotechnology 2016;100:2141-2151.

59. Solovieva EV, Myakinina VP, Kislichkina AA et al. Comparative genome analysis of novel Podoviruses lytic for hypermucoviscous Klebsiella pneumoniae of K1, K2, and K57 capsular types. Virus Res 2018;243:10-18.

60. Kleiner M, Hooper LV, Duerkop BA. Evaluation of methods to purify virus-like particles for metagenomic sequencing of intestinal viromes. BMC genomics 2015;16:7.

61. Petrie KL, Palmer ND, Johnson DT et al. Destabilizing mutations encode nongenetic variation that drives evolutionary innovation. Science 2018;359:1542-1545.

62. Roux S, Enault F, Hurwitz BL et al. VirSorter: mining viral signal from microbial genomic data. PeerJ 2015;3:e985.

63. Sandmeler $\mathrm{H}$. Acquisition and rearrangement of sequence motifs in the evolution of bacteriophage tail fibres. Molecular microbiology 1994;12:343-350.

64. Nobrega FL, Vlot $\mathrm{M}$, de Jonge PA et al. Targeting mechanisms of tailed bacteriophages. Nature Reviews Microbiology 2018;16:760-773.

65. Jensen EC, Schrader HS, Rieland B et al. Prevalence of Broad-Host-Range Lytic Bacteriophages of Sphaerotilus natans, Escherichia coli, andPseudomonas aeruginosa. Applied and environmental microbiology 1998;64:575-580.

66. Townsend E, Moat J, Jameson E. CAUTI's Next Top Model - model dependent Klebsiella biofilm inhibition by bacteriophages and antimicrobials. bioRxiv 2020:2020.2006.2030.179804.

67. March C, Cano V, Moranta D et al. Role of bacterial surface structures on the interaction of Klebsiella pneumoniae with phagocytes. PloS one 2013;8.

68. Whitfield C. Biosynthesis and assembly of capsular polysaccharides in Escherichia coli. Annu Rev Biochem 2006;75:39-68.

69. Simoons-Smit A, Verweij-van Vught A, MacLaren D. The role of $K$ antigens as virulence factors in Klebsiella. Journal of medical microbiology 1986;21:133-137.

70. Edwards P, Fife MA. Capsule types of Klebsiella. The Journal of infectious diseases 1952:92-104.

71. Ørskov I. Serological Investigations in the Klebsiella Group. 1. New Capsule Types. Jeta Path et Microb Scandinavica 1955;36:449-453.

72. Choby JE, Howard-Anderson J, Weiss DS. Hypervirulent Klebsiella pneumoniaeclinical and molecular perspectives. Journal of internal medicine 2020;287:283-300.

73. Hsu C-R, Lin T-L, Pan Y-J et al. Isolation of a bacteriophage specific for a new capsular type of Klebsiella pneumoniae and characterization of its polysaccharide depolymerase. PloS one $2013 ; 8$.

74. Bhetwal A, Maharjan A, Shakya S et al. Isolation of Potential Phages against Multidrug-Resistant Bacterial Isolates: Promising Agents in the Rivers of Kathmandu, Nepal. Biomed Res Int 2017;2017:3723254. 
75. Hoyles L, Murphy J, Neve H et al. Klebsiella pneumoniae subsp. pneumoniaebacteriophage combination from the caecal effluent of a healthy woman. PeerJ 2015;3:e1061.

76. Rieger-Hug D, Stirm S. Comparative study of host capsule depolymerases associated with Klebsiella bacteriophages. Virology 1981;113:363-378.

77. Hughes K, Sutherland I, Clark J et al. Bacteriophage and associated polysaccharide depolymerases-novel tools for study of bacterial biofilms. Journal of applied microbiology 1998;85:583-590.

78. Pan YJ, Lin TL, Chen CC et al. Klebsiella Phage PhiK64-1 Encodes Multiple Depolymerases for Multiple Host Capsular Types. J Virol 2017;91:e02457-02416.

79. Kakasis A, Panitsa G. Bacteriophage therapy as an alternative treatment for human infections. A comprehensive review. International journal of antimicrobial agents 2019;53:16-21.

80. Chan BK, Abedon ST. Phage therapy pharmacology: phage cocktails. In: Advances in applied microbiology. (Elsevier). 2012; pp. 1-23.

81. Chan BK, Abedon ST, Loc-Carrillo C. Phage cocktails and the future of phage therapy. Future Microbiol 2013;8:769-783.

82. Nale JY, Spencer J, Hargreaves KR et al. Bacteriophage combinations significantly reduce Clostridium difficile growth in vitro and proliferation in vivo. Antimicrobial agents and chemotherapy 2016;60:968-981.

83. Van Laar TA, Chen T, You T et al. Sublethal concentrations of carbapenems alter cell morphology and genomic expression of Klebsiella pneumoniae biofilms. Antimicrobial agents and chemotherapy 2015;59:1707-1717.

84. Ranieri MR, Whitchurch CB, Burrows LL. Mechanisms of biofilm stimulation by subinhibitory concentrations of antimicrobials. Current opinion in microbiology 2018;45:164-169.

85. CHATAIN-LY MH. The factors affecting effectiveness of treatment in phages therapy. Frontiers in microbiology 2014;5:51.

86. Gill JJ, Hyman P. Phage choice, isolation, and preparation for phage therapy. Current pharmaceutical biotechnology 2010;11:2-14.

87. Dąbrowska K, Miernikiewicz P, Piotrowicz A et al. Immunogenicity Studies of Proteins Forming the T4 Phage Head Surface. Journal of Virology 2014;88:12551. 
bioRxiv preprint doi: https://doi.org/10.1101/2020.07.05.179689; this version posted November 24,2020 . The copyright holder for this preprint (which was not certified by peer review) is the author/funder, who has granted bioRxiv a license to display the preprint in perpetuity. It is made available under aCC-BY-NC 4.0 International license.

Table 1. Details of Klebsiella species and strains used in this study. Capsule types are given where applicable, alongside the origin of the strain and indication of use as an isolation host.

\begin{tabular}{|c|c|c|c|c|c|}
\hline Species & Strain & $\begin{array}{l}\text { Capsule } \\
\text { Type }\end{array}$ & $\begin{array}{l}\text { Isolation } \\
\text { Host? }\end{array}$ & Origin & Isolation source \\
\hline Klebsiella aerogenes & 30053 & - & $\checkmark$ & $\begin{array}{l}\text { DSMZ Culture } \\
\text { Collection }\end{array}$ & sputum \\
\hline Klebsiella michiganensis & 25444 & 01v1 & $x$ & $\begin{array}{l}\text { DSMZ Culture } \\
\text { Collection }\end{array}$ & toothbrush holder \\
\hline \multirow{5}{*}{ Klebsiella oxytoca } & \multicolumn{2}{|c|}{$17074801 v 1$} & $x$ & Clinical Isolate & $\begin{array}{l}\text { catheter specimen } \\
\text { urine }\end{array}$ \\
\hline & 5175 & KL29 & $x$ & $\begin{array}{l}\text { DSMZ Culture } \\
\text { Collection }\end{array}$ & pharyngeal tonsil \\
\hline & 25736 & KL74 & $\checkmark$ & $\begin{array}{l}\text { DSMZ Culture } \\
\text { Collection }\end{array}$ & case of pneumonia \\
\hline & 170821 & 1OL104 & $\checkmark$ & Clinical Isolate & Urine \\
\hline & 171266 & 6OL104 & $x$ & Clinical Isolate & urostomy urine \\
\hline \multirow{13}{*}{ Klebsiella pneumoniae } & 170958 & $8 K L 28$ & $\checkmark$ & Clinical Isolate & urine \\
\hline & 171304 & 4KL144 & $x$ & Clinical Isolate & $\begin{array}{l}\text { catheter specimen } \\
\text { urine }\end{array}$ \\
\hline & 13440 & KL38 & $\checkmark$ & $\begin{array}{l}\text { NCTC Culture } \\
\text { Collection }\end{array}$ & clinical \\
\hline & 13442 & KL110 & $x$ & $\begin{array}{l}\text { NCTC Culture } \\
\text { Collection }\end{array}$ & hospital, Italy \\
\hline & 30104 & KL3 & $\checkmark$ & $\begin{array}{l}\text { DSMZ Culture } \\
\text { Collection }\end{array}$ & human blood \\
\hline & 13465 & KL57 & $x$ & $\begin{array}{l}\text { NCTC Culture } \\
\text { Collection }\end{array}$ & clinical \\
\hline & 170820 & OKL158 & $x$ & Clinical Isolate & urine \\
\hline & 16358 & KL4 & $x$ & $\begin{array}{l}\text { DSMZ Culture } \\
\text { Collection }\end{array}$ & human, nose \\
\hline & 170723 & $3 \mathrm{KL} 2$ & $\checkmark$ & Clinical Isolate & urine \\
\hline & 171167 & $7 \mathrm{KL} 2$ & $x$ & Clinical Isolate & urine \\
\hline & 13443 & $\mathrm{KL} 2$ & $x$ & $\begin{array}{l}\text { NCTC Culture } \\
\text { Collection }\end{array}$ & clinical \\
\hline & 13882 & KL64 & $x$ & $\begin{array}{l}\text { ATCC Culture } \\
\text { Collection }\end{array}$ & water \\
\hline & 13439 & KL14 & $\checkmark$ & $\begin{array}{l}\text { NCTC Culture } \\
\text { Collection }\end{array}$ & outbreak strain \\
\hline \multirow[b]{2}{*}{ Klebsiella variicola } & W12 & KL14 & $x$ & Environmental Isolate & soil \\
\hline & 15968 & KL16 & $\checkmark$ & $\begin{array}{l}\text { DSMZ Culture } \\
\text { Collection }\end{array}$ & banana root \\
\hline \multirow{2}{*}{$\begin{array}{l}\text { Klebsiella } \\
\text { quasipneumoniae }\end{array}$} & 28211 & KL35 & $\checkmark$ & $\begin{array}{l}\text { DSMZ Culture } \\
\text { Collection }\end{array}$ & human blood \\
\hline & 700603 & $3 \mathrm{KL53}$ & $\checkmark$ & $\begin{array}{l}\text { ATCC Culture } \\
\text { Collection }\end{array}$ & urine \\
\hline
\end{tabular}

Table 2. Phage isolate details. Lab ID refers to the laboratory identification number, source of isolation, indicates where the water sample was collected for phage enrichment and isolation and the strain of isolation indicates Klebsiella sp. strain on which 3 rounds of plaque assay isolation were performed. Accession numbers refers to associated project accession numbers assigned by the ENA for each phage. 
bioRxiv preprint doi: https://doi.org/10.1101/2020.07.05.179689; this version posted November 24,2020 . The copyright holder for this preprint (which was not certified by peer review) is the author/funder, who has granted bioRxiv a license to display the preprint in perpetuity. It is made available under aCC-BY-NC 4.0 International license.

\begin{tabular}{|c|c|c|c|c|c|}
\hline Phage Name & $\begin{array}{l}\text { Lab } \\
\text { ID }\end{array}$ & Source of isolation & & Strain of isolation & $\begin{array}{l}\text { Accession } \\
\text { number }\end{array}$ \\
\hline Klebsiella phage vB_KaS-Benoit & 1 & Estuary & Jelitkowo, Poland & Klebsiella aerogenes DSM 30053 & PRJEB39773 \\
\hline Klebsiella phage VB_KaS-Veronica & 2 & Marine canal & Grand canal, Venice, Italy & Klebsiella aerogenes DSM 30053 & PRJEB40165 \\
\hline Klebsiella phage vB_KvM-Eowyn & 4 & Estuary & Jelitkowo, Poland & Klebsiella variicola DSM 15968 & PRJEB40131 \\
\hline Klebsiella phage vB_KaS-Gatomon & 6 & Marine canal & Grand canal, Venice, Italy & Klebsiella aerogenes DSM 30053 & PRJEB40159 \\
\hline Klebsiella phage vB_KaS-Ahsoka & 7 & Slurry & Slurry tank, UK & Klebsiella aerogenes DSM 30053 & PRJEB40160 \\
\hline Klebsiella phage vB_KppS-Samwise & 8 & Slurry & Slurry tank, UK & Klebsiella pneumoniae DSM 30104 & PRJEB40161 \\
\hline Klebsiella phage vB_KppS-Totoro & 10 & Estuary & Jelitkowo, Poland & Klebsiella pneumoniae DSM 30104 & PRJEB40166 \\
\hline Klebsiella phage vB_KoM-Pickle & 12 & Estuary & Jelitkowo, Poland & Klebsiella oxytoca DSM 25736 & PRJEB40176 \\
\hline Klebsiella phage vB_KppS-Ponyo & 19 & River & Gneiwkowo, Poland & Klebsiella pneumoniae DSM 30104 & PRJEB40167 \\
\hline Klebsiella phage vB_KppS-Jiji & 27 & Pond & Gneiwkowo, Poland & Klebsiella pneumoniae DSM 30104 & PRJEB40168 \\
\hline Klebsiella phage vB_KppS-Raw & 33 & Sewage - raw & Spernal sewage works, UK & Klebsiella pneumoniae DSM 30104 & PRJEB40132 \\
\hline Klebsiella phage vB_KppS-Storm & 34 & Sewage - storm tank & Spernal sewage works, UK & Klebsiella pneumoniae DSM 30104 & PRJEB40169 \\
\hline Klebsiella phage vB_KppS-Ant & 35 & Sewage - anoxic sludge & Spernal sewage works, UK & Klebsiella pneumoniae DSM 30104 & PRJEB40148 \\
\hline Klebsiella phage vB_KqM-LilBean & 36 & Sewage - raw & Spernal sewage works, UK & Klebsiella quasipneumoniae DSM 28211 & PRJEB40171 \\
\hline Klebsiella phage vB_KqM-Bilbo & 38 & Sewage - raw & Spernal sewage works, UK & Klebsiella quasipneumoniae DSM 28211 & PRJEB40172 \\
\hline Klebsiella phage vB_KqM-Westerburg & 39 & Sewage - raw & Spernal sewage works, UK & Klebsiella quasipneumoniae DSM 28211 & PRJEB40173 \\
\hline Klebsiella phage vB_KpP-Yoda & 43 & Sewage - storm tank & Spernal sewage works, UK & Klebsiella pneumoniae 170723 & PRJEB40162 \\
\hline Klebsiella phage vB_KqP-Goliath & 44 & Sewage - raw & Spernal sewage works, UK & Klebsiella quasipneumoniae DSM 700603 & PRJEB40163 \\
\hline Klebsiella phage vB_KpP-Screen & 46 & Sewage - sieve & Spernal sewage works, UK & Klebsiella pneumoniae 170723 & PRJEB40164 \\
\hline Klebsiella phage vB_KppS-Eggy & 49 & Sewage - anoxic sludge & Spernal sewage works, UK & Klebsiella pneumoniae DSM 30104 & PRJEB40146 \\
\hline Klebsiella phage vB_KppS-Pokey & 50 & Sewage - anoxic sludge & Spernal sewage works, UK & Klebsiella pneumoniae DSM 30104 & PRJEB40147 \\
\hline Klebsiella phage vB_KppS-Anoxic & 52 & Sewage - anoxic sludge & Spernal sewage works, UK & Klebsiella pneumoniae DSM 30104 & PRJEB40170 \\
\hline Klebsiella phage vB_KoM-Liquor & 61 & Sewage - mixed liquor & Spernal sewage works, UK & Klebsiella oxytoca 170821 & PRJEB40174 \\
\hline Klebsiella phage vB_KpM-Milk & 62 & Sewage - mixed liquor & Spernal sewage works, UK & Klebsiella oxytoca 170821 & PRJEB40175 \\
\hline Klebsiella phage vB_KoM-Flushed & 63 & Sewage - mixed liquor & Spernal sewage works, UK & Klebsiella pneumoniae 170958 & PRJEB40181 \\
\hline Klebsiella phage vB_KpM-Wobble & 64 & Sewage - mixed liquor & Spernal sewage works, UK & Klebsiella pneum & PRJEB40182 \\
\hline Klebsiella phage vB_KpM-Mild & 65 & Sewage - mixed liquor & Spernal sewage works, UK & Klebsiella pneumoniae DSM 13439 & PRJEB40177 \\
\hline Klebsiella phage vB_KpM-KalD & 67 & Sewage - mixed liquor & Spernal sewage works, UK & Klebsiella pneumoniae DSM 13439 & PRJEB40178 \\
\hline Klebsiella phage vB_KoM-MeTiny & 68 & Sewage - mixed liquor & Spernal sewage works, UK & Klebsiella oxytoca DSM 25736 & PRJEB40179 \\
\hline Klebsiella phage vB_KpM-SoFaint & 70 & Sewage - mixed liquor & Spernal sewage works, UK & Klebsiella pneumoniae DSM 13440 & PRJEB40180 \\
\hline
\end{tabular}


bioRxiv preprint doi: https://doi.org/10.1101/2020.07.05.179689; this version posted November 24, 2020. The copyright holder for this preprint (which was not certified by peer review) is the author/funder, who has granted bioRxiv a license to display the preprint in perpetuity. It is made available under aCC-BY-NC 4.0 International license.

Table 3. Phage taxonomy and similarity to closest sequenced phage. Family, Subfamily and Genus are assigned based on the clustering patterns observed in vConTACT2 analysis (fig. 2), and conserved branching patterns observed in the marker gene phylogenetic trees (fig. S12-20). ANI refers to Average Nucleotide Identity calculated with orthoANI (https://www.ezbiocloud.net/tools/ani). Details for "comparison phage" relate to details of previously sequenced phages available in public databases used for ANI analysis.

\begin{tabular}{|c|c|c|c|c|c|c|c|c|}
\hline Phage name & $\begin{array}{l}\text { Lab } \\
\text { ID }\end{array}$ & $\begin{array}{l}\text { Phage } \\
\text { group }\end{array}$ & Taxonomy & Subfamily & Genus & $\begin{array}{l}\text { Comparison phage } \\
\text { Phage name }\end{array}$ & Accession & ANI \\
\hline vB_KppS-Raw & 33 & A & Siphoviridae & & Nonagvirus & Enterobacteria phage JenP2 & KP719132 & 66.77 \\
\hline vB_KppS-Eggy & 49 & A & Siphoviridae & & Nonagvirus & Enterobacteria phage JenP2 & KP719132 & 65.86 \\
\hline VB_KppS-Pokey & 50 & A & Siphoviridae & & Nonagvirus & Enterobacteria phage Jen $\mathrm{P} 2$ & KP719132 & 66.69 \\
\hline vB_KppS-Ant & 35 & B & Unclassified & & & CCaudovirales_phage_clone_3F_1 & MF417951 & 90.14 \\
\hline vB_KaS-Gatomon & 6 & c & Drexlenviridae & Tempevirinae & unclassified & Escherichia phage Henu7 & MN019128 & 93.55 \\
\hline vB_KaS-Ahsoka & 7 & c & Drexlerviridae & Tempevirinae & unclassified & scherichia phage Henu7 & MN019128 & 92.09 \\
\hline vB_KppS-Samwise & 8 & c & Drexlerviridae & Tempevirinae & unclassified & Escherichia phage Henu7 & MN019128 & 93.55 \\
\hline vB_KvM-Eowyn & 4 & D & Myoviridae & unclassified & & Serratia_phage_KpHz_2 & KF806589 & 95.02 \\
\hline vB_KpP-Yoda & 43 & $\mathrm{E}$ & Autographiviridae & Slopekvirinae & Drulisvirus & Klebsiella phage vB_KpnP_SU552A & KP708986 & 87.03 \\
\hline VB_KqP-Goliath & 44 & $\mathrm{E}$ & Autographiviridae & Slopekvirinae & Drulisvirus & Klebsiella phage vB_KpnP_SU552A & KP708986 & 85.82 \\
\hline VB_KpP-Screen & 46 & $\mathrm{E}$ & Autographiviridae & Slopekvirinae & Drulisvirus & Klebsiella phage vB_KpnP_SU552A & KP708986 & 86.58 \\
\hline vB_KaS-Benoit & 1 & $\mathrm{~F}$ & Demerecviridae & & Sugarlandvirus & VB_Kn_|ME260 & NC_041899 & 94.29 \\
\hline vB_KaS-Veronica & 2 & $\mathrm{~F}$ & Demerecviridae & & Sugarlandvirus & 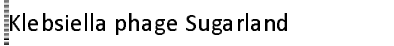 & NC_042093 & 93.46 \\
\hline vB_KppS-Totoro & 10 & $\mathrm{~F}$ & Demerecviridae & & Sugarlandvirus & EB_Kpn_IME260 & NC_041899 & 94.29 \\
\hline VB_KppS-Ponyo & 19 & $\mathrm{~F}$ & Demerecviridae & & Sugarlandvirus & Klebsiella phage Sugarland & NC_042093 & 94.15 \\
\hline vB_KppS-Jiji & 27 & $\mathrm{~F}$ & Demerecviridae & & Sugarlandvirus & Klebsiella phage Sugarland & NC_042093 & 93.26 \\
\hline VB_KppS-Storm & 34 & $\mathrm{~F}$ & Demerecviridae & & Sugarlandvirus & Klebsiella phage Sugarland & NC_042093 & 95.02 \\
\hline VB_KppS-Anoxic & 52 & $\mathrm{~F}$ & Demerecviridae & & Sugarlandvirus & NB_Kpn_IME260 & NC_041899 & 96.11 \\
\hline vB_KqM-LilBean & 36 & G & Ackermannviridae & & Taipeivirus & Klebsiella virus 0507 KN21 & NC_022343 & 97.83 \\
\hline $\begin{array}{l}\text { vB_KqM-Bilbo } \\
\text { vB_KqM- }\end{array}$ & 38 & G & Ackermannviridae & & Taipeivirus & Klebsiella virus 0507 KN21 & NC_022343 & 97.22 \\
\hline Westerburg & 39 & G & Ackermannviridae & & Taipeivirus & Klebsiella virus 0507 KN21 & NC_022343 & 96.93 \\
\hline vB_KoM-Pickle & 12 & $\mathrm{H}$ & Myoviridae & Tevenvirinae & Slopekvirus & Klebsiella phage KP15 & GU295964 & 97.93 \\
\hline vB_KoM-Liquor & 61 & H & Myoviridae & Tevenvirinae & Slopekvirus & 䜌lebsiella phage KP15 & GU295964 & 97.83 \\
\hline vB_KpM-Milk & 62 & $\mathrm{H}$ & Myoviridae & Tevenvirinae & Slopekvirus & 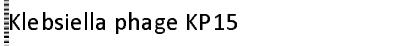 & GU295964 & 98.38 \\
\hline vB_KpM-Mild & 65 & H & Myoviridae & Tevenvirinae & Slopekvirus & 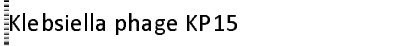 & GU295964 & 97.65 \\
\hline vB_KpM-KalD & 67 & $\mathrm{H}$ & Myoviridae & Tevenvirinae & Slopekvirus & Klebsiella phage KP15 & GU295964 & 97.42 \\
\hline VB_KoM-MeTiny & 68 & H & Myoviridae & Tevenvirinae & Slopekvirus & Klebsiella phage KP15 & GU295964 & 97.92 \\
\hline vB_KpM-SoFaint & 70 & H & Myoviridae & Tevenvirinae & Slopekvirus & Klebsiella phage KP15 & GU295964 & 97.72 \\
\hline vB_KoM-Flushed & 63 & I & Myoviridae & Tevenvirinae & Jiaodavirus & 翯lebsiella phage JD18 & KT239446 & 96.19 \\
\hline vB_KpM-Wobble & 64 & I & Myoviridae & Tevenvirinae & Jiaodavirus & 竐lebsiella phage JD18 & KT239446 & 97.01 \\
\hline
\end{tabular}


bioRxiv preprint doi: $h t t p s: / / d o i . o r g / 10.1101 / 2020.07 .05 .179689$; this version posted November 24,2020 . The copyright holder for this preprint (which was not certified by peer review) is the author/funder, who has granted bioRxiv a license to display the preprint in perpetuity. It is made available under aCC-BY-NC 4.0 International license.

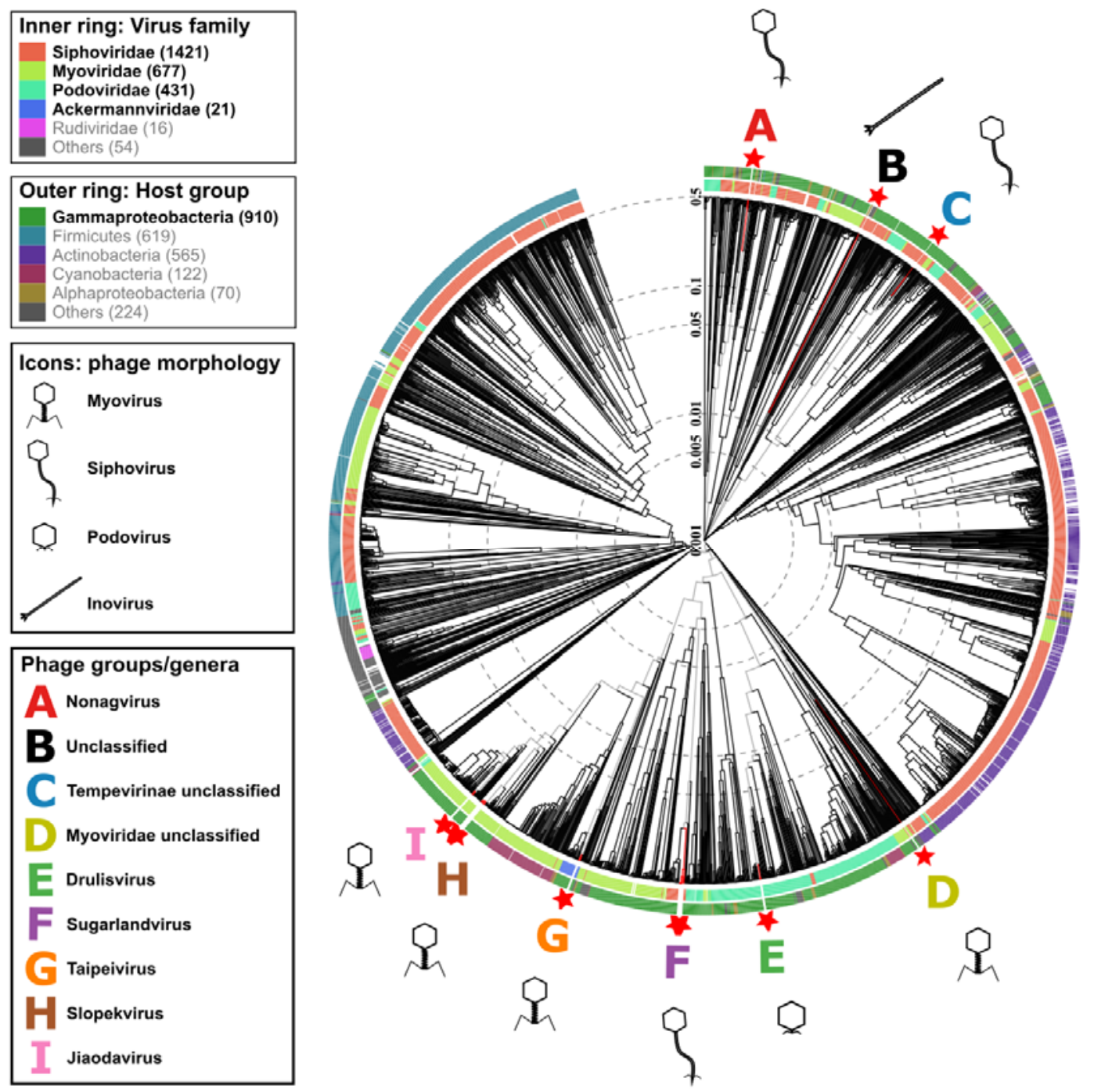

Figure 1. Protein level phylogenetic tree, generated by VIPtree. Klebsiella phage isolates (囫). A-I denote phage groups with the following genera: A. Nonagvirus; B. unclassified family/genus; C. Tempevirinae unclassified; D. Myoviridae unclassified; E. Drulisvirus; F. Sugarlandvirus; G.

Taipeivirus; H. Slopekvirus and I. Jiaodavirus. Icons indicate phage morphology. 


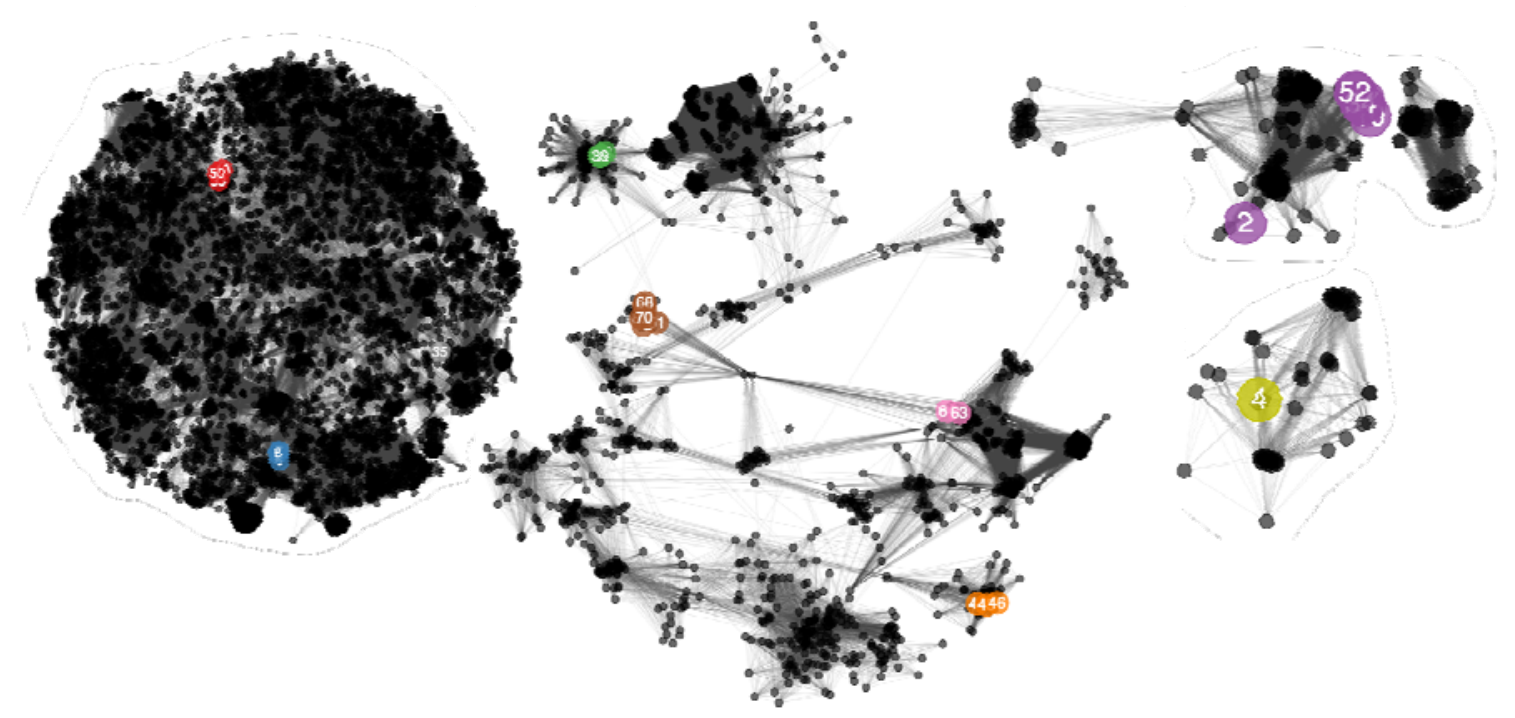

Figure 2. Network analysis of phage-encoded proteins calculated with vConTACT2. Coloured, numbered nodes represent our Klebsiella phage isolates, coloured according to the phage group and subsequent genera to which each phage belongs. Numbers within nodes indicate the lab identification numbers (see table 2). Smaller, black nodes represent previously sequenced phages as references. Edges between nodes represent shared proteins, such that many connecting edges implies greater pairwise shared protein content. Phage nodes are clustered based on shared proteins, with a springembedded (force directed) layout visualisation created in Cytoscape. 
bioRxiv preprint doi: https://doi.org/10.1101/2020.07.05.179689; this version posted November 24,2020 . The copyright holder for this preprint (which was not certified by peer review) is the author/funder, who has granted bioRxiv a license to display the preprint in perpetuity. It is made available under aCC-BY-NC 4.0 International license.

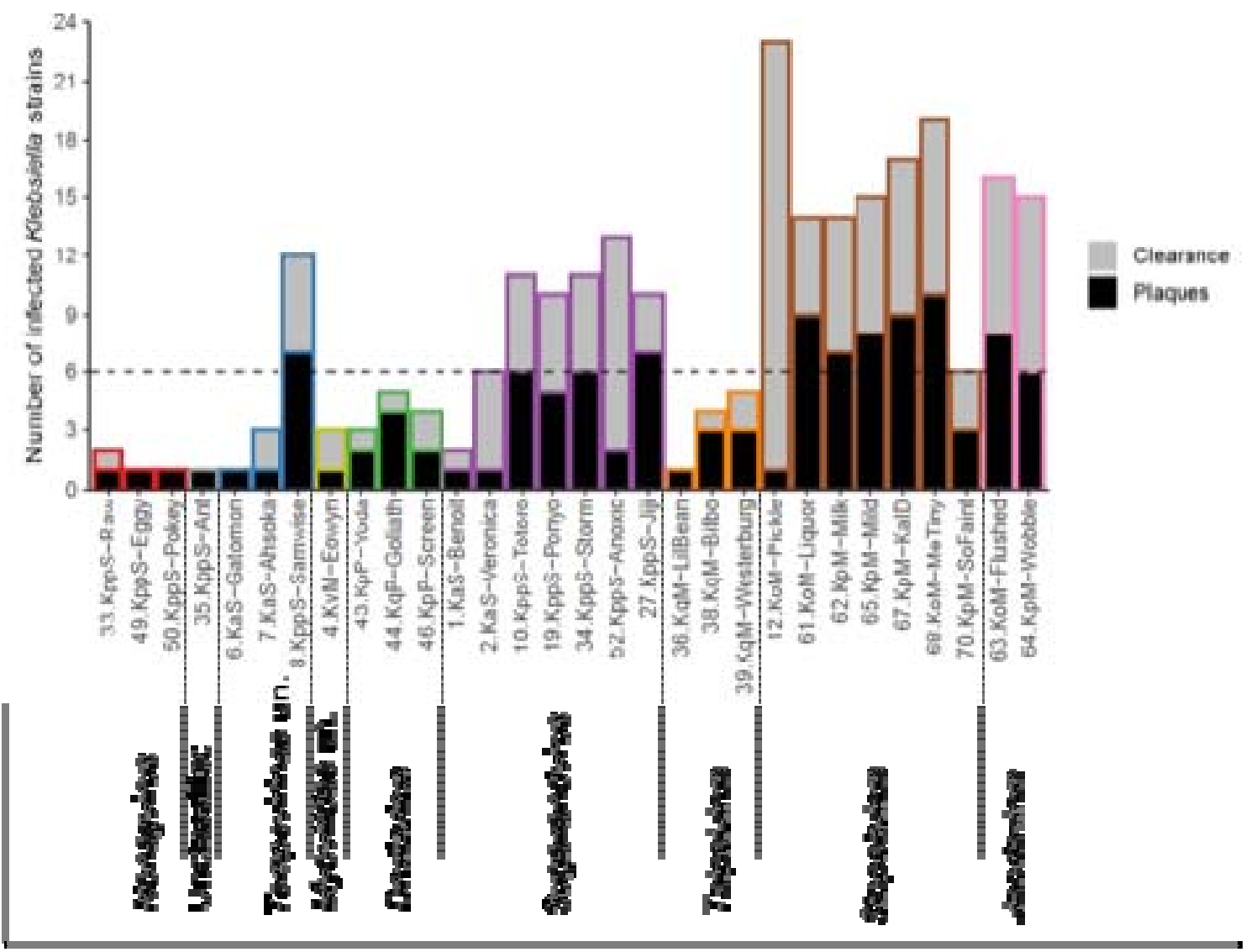

Figure 3. Number of Klebsiella strains infected by each bacteriophage out of a possible 24. Plaques, indicates the number of strains a phage replicated in and resulted in plaques on overlay agar; Clearance, indicates the additional number of strains the phage cleared or partially cleared the bacterial lawn during spot testing analysis (Figure S1). Totals include the isolation host. Bars marked with \# denote an incomplete data set. Coloured outlines relate to the phage groups, as identified in figure 1 , with genera annotated for each group. 
bioRxiv preprint doi: https://doi.org/10.1101/2020.07.05.179689; this version posted November 24, 2020. The copyright holder for this preprint (which was not certified by peer review) is the author/funder, who has granted bioRxiv a license to display the preprint in perpetuity. It is made available under aCC-BY-NC 4.0 International license.

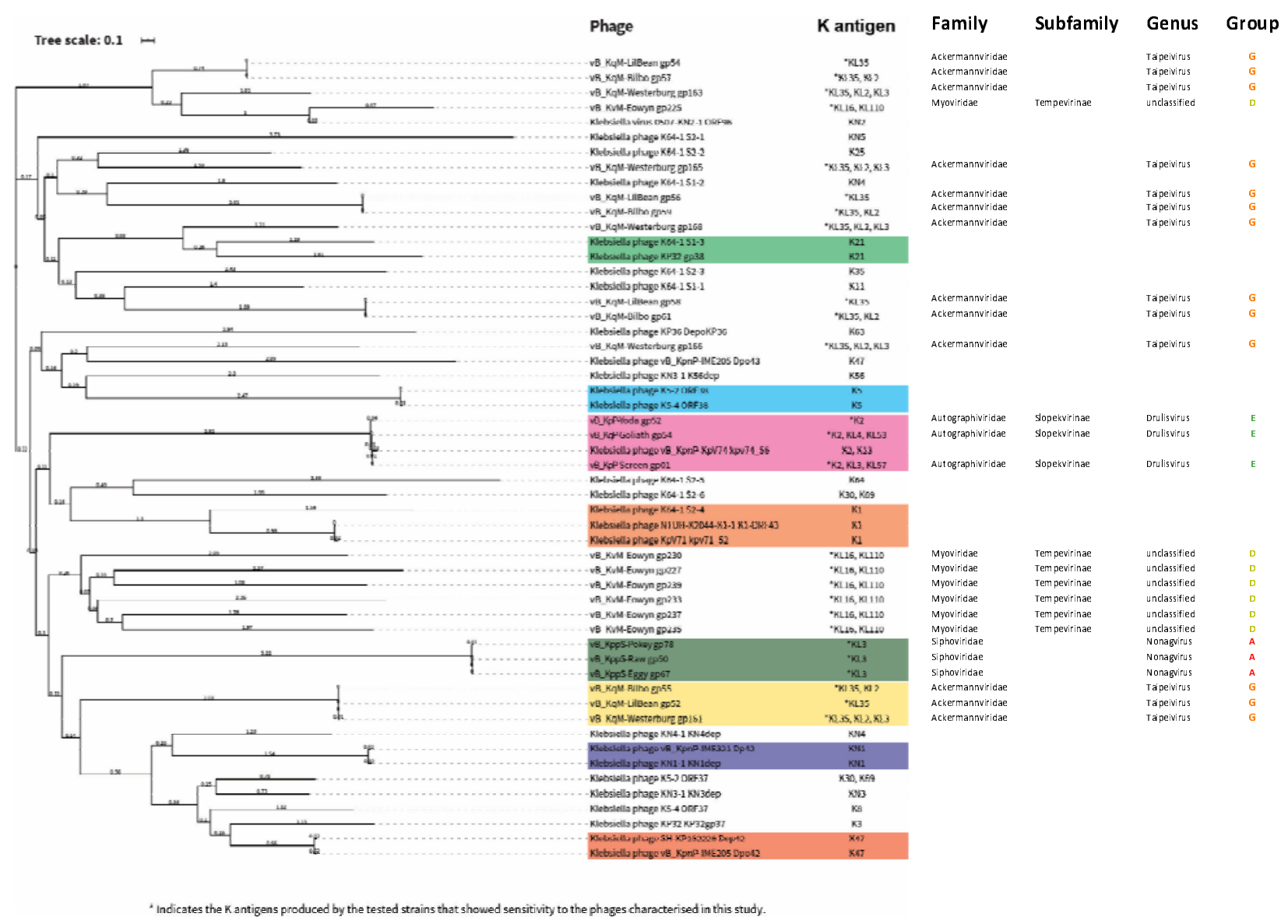

Figure 4. Phylogenetic tree of previously biochemically characterised depolymerase proteins that target Klebsiella spp. and putative depolymerase proteins in our phage isolates. Sequences were aligned with Muscle using SeaView. Phylogenetic tree construction was performed with MegaX with 500 bootstrap calculations using the LG model. Tree topology searches were performed using a combination of $\mathrm{NNI}$ and $\mathrm{NJ} /$ BioNJ. The tree was subsequently visualised and annotated using iTOL (v4). Depolymerases highlighted in colour blocks have overlapping potential target $\mathrm{K}$ antigens. 
bioRxiv preprint doi: https://doi.org/10.1101/2020.07.05.179689; this version posted November 24,2020 . The copyright holder for this preprint (which was not certified by peer review) is the author/funder, who has granted bioRxiv a license to display the preprint in perpetuity. It is made available under aCC-BY-NC 4.0 International license.

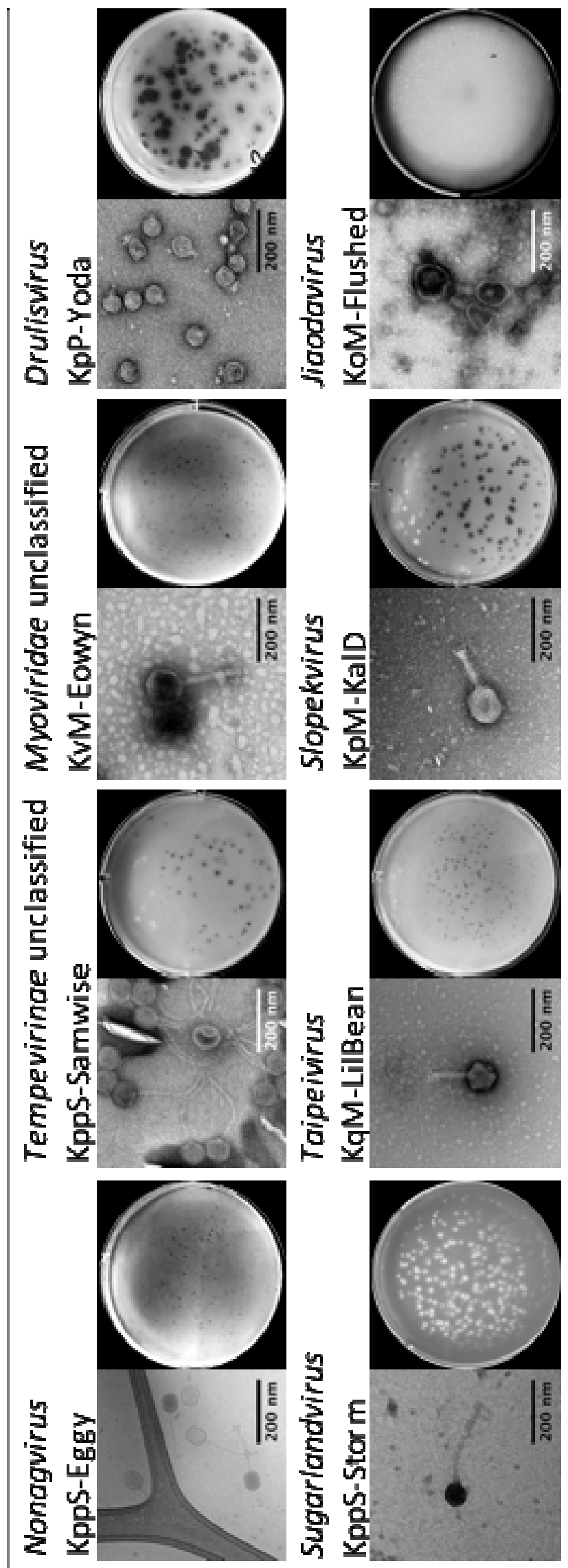

Figure 5. Phage morphology, showing representative TEM and plaque images for each phage group. TEM and plaque assays were performed as described in the methods. Scale bars on the TEM images represent $200 \mathrm{~nm}$. Further images of all phages can be found in the supplementary materials, figure s? 
bioRxiv preprint doi: https://doi.org/10.1101/2020.07.05.179689; this version posted November 24, 2020. The copyright holder for this preprint (which was not certified by peer review) is the author/funder, who has granted bioRxiv a license to display the preprint in perpetuity. It is made A
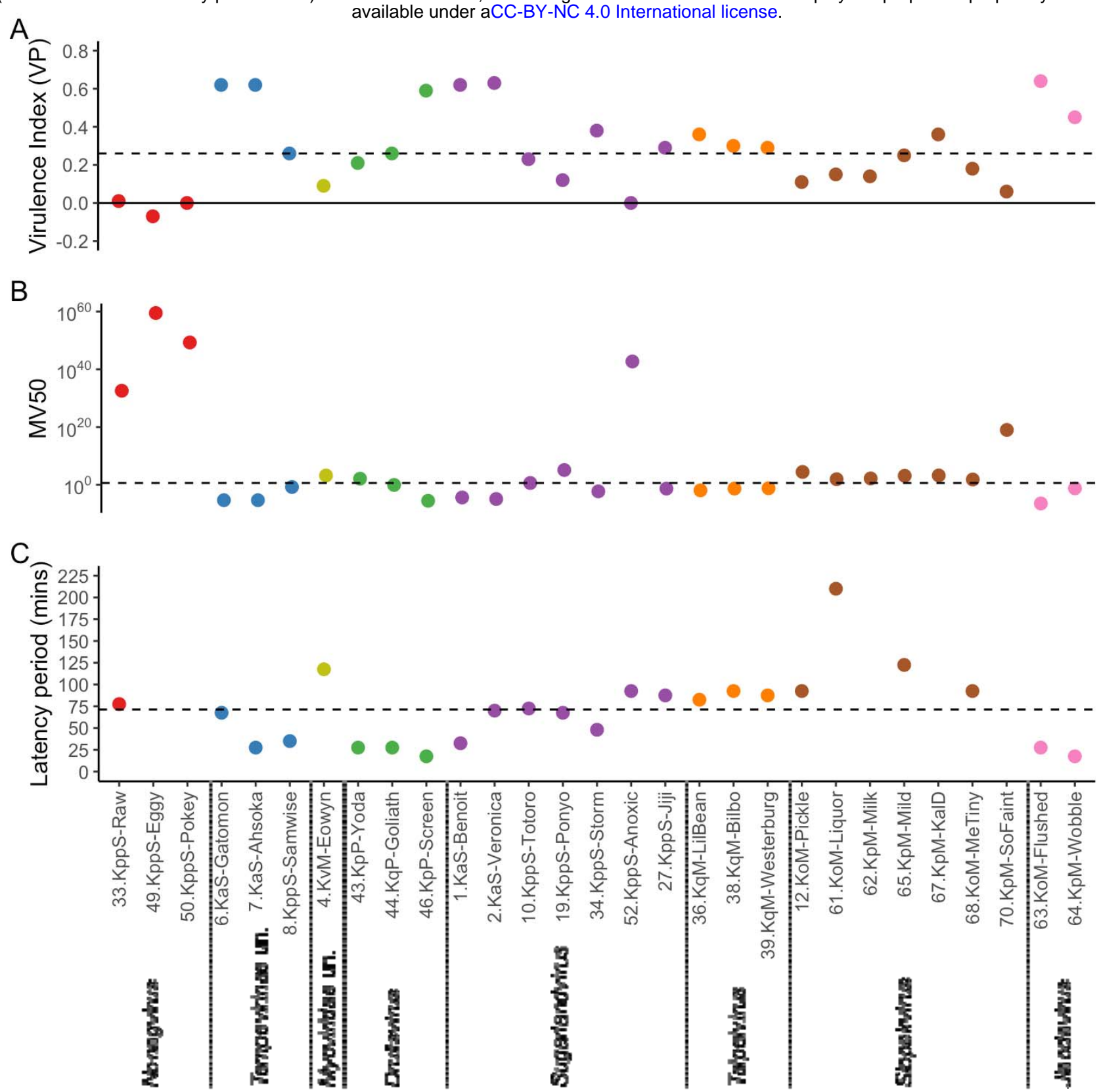

Figure 6. Lysis period and virulence indices are not correlated, but temperate phages are less virulent. Panel $(A)$ displays the virulence index of the phages. Virulence index is a quantified measure of the phage, in their respective isolation host (table 2), in LB with $5 \mathrm{mM}$ each of $\mathrm{CaCl}_{2}$ and $\mathrm{MgCl}_{2}$, at $37 \mathrm{C}$. Panel (B) displays the MV50, the MOI at which each phage achieves $50 \%$ of their maximal theoretical virulence. Both of these virulence measures are described in more detail by Storms ${ }^{47}$. Panel (C) displays the lysis period of the phage. Where this is left blank, a lysis period could not be established usually indicating temperate lifestyle. Coloured outlines relate to the phage groups, as identified in figure 1, with genera annotated for each group. Dashed lines indicate the median values for each metric. 